\title{
Dual-color optical activation and suppression of neurons with high temporal precision
}

Noëmie Mermet-Joret ${ }^{1,2,3}$, Andrea Moreno ${ }^{1,2,3}$, Agnieszka Zbela ${ }^{4}$, Bárður Eyjólfsson Ellendersen ${ }^{1,2}$, Nathalie Krauth ${ }^{1,2,3, \dagger}$, Anne von Philipsborn ${ }^{1,2}$, Joaquin Piriz ${ }^{5}$, John Y. Lin ${ }^{4,6, *}$ and Sadegh Nabavi ${ }^{1,2,3,6, *}$

A fundamental challenge in the optogenetic toolbox is that all opsins, regardless of their excitation spectra, are activated by blue light. We hypothesized that pairing a red-shifted channelrhodopsin with a blue light-sensitive anion channel of appropriately matching kinetics shall render neurons responsive to a red but not blue light. To achieve this, we used a semi-rational mutagenesis strategy to optimize the kinetics and light spectrum of a chloride channelrhodopsin. By pairing optimized variants of blue light-sensitive anion channel ZipACR with vfChrimson, a fast red-shifted channelrhodopsin, we created a system in which red light derives precise and faithful action potentials of high frequencies, while blue light, through shunting inhibition, nullifies the effect of the red-shifted ChR2. Additionally, by a simple switch between red and blue lights, one can effectively excite or inhibit the activity of the same neurons.

Despite the short history of optogenetics, optical manipulation of neuronal activity has become an indispensable tool in the studies for functional analysis of brain circuits. The increasing needs for a more precise temporal and spatial manipulation of the neurons along with the untapped potentials of optogenetics have been driving forces in expanding the repertoire of optogenetics toolbox. Main development among these expansions is the discovery of opsins of diverse excitation spectra, with the current excitatory opsins showing light sensitivity ranging from near UV to near far-red spectra (Yizhar et al., 2011; Deisseroth and Hegemann, 2017; Marshel et al., 2019). The main purpose for such developments is to independently as well as collectively control the activity of distinct populations of neurons. This aim, however, has not been satisfied, mainly because all opsins, in addition to their own excitation spectral peak, are activated by blue light, a problem known in the field as cross-over or bleed-through. Attempts to create blue light-insensitive opsins will be most likely unsuccessful due to the intrinsic blue absorption by the retinal molecule, the non-encoded chromophore, which captures light energy in channelrhodopsins (Waddell et al., 1973; Wang et al., 2012).

There have been two stand out attempts to overcome the problem of cross-over. One uses a variant of blue-shifted ChR2 with fast kinetics in combination with a slow kinetics red-shifted ChR2 (R-ChR) (Klapoetke et al., 2014). The reasoning is that short pulses of blue light will activate only fast ChR2. Since it requires a tight regulation of the protein expression as well as the light intensity, this method has not been adopted for in-vivo application. Another approach is to expose axons expressing a R-ChR to prolonged photostimulation. Immediately following this, axons be- come unresponsive to light pulses and cease to fire. This method is limited to axonal regions as prolonged optical stimulation of the soma induces trains of action potentials (Hooks et al., 2015).

A potential solution to the problem of cross-over, the one that we propose here, is to co-express a blue-shifted anion channelrhodopsin (B-ACR) along with the R-ChR to 'suppress' its excitation by blue light. For this purpose, we chose vfChrimson- currently the fastest R-ChR- to gain more precise temporal control of red light excitation (Mager et al., 2018). This would, however, require a new variant of anion channelrhodopsin to optimize pairing. We used a semi-rational mutagenesis approach to modify the kinetic properties of ZipACR, an ultrafast blue light-activated chloride channel (Govorunova et al., 2017). We describe here two variants of ZipACR, I151T and I151V, with appropriate kinetics, light spectra, and photocurrent. We then paired the optimized anion channels with vfChrimson in a single expression unit. The new system, Zip-IvfChr, can drive time-locked high-frequency action potentials (APs) in response to red pulses $(635 \mathrm{~nm})$. In response to blue pulses ( $470 \mathrm{~nm}$ ), on the other hand, Zip-IvfChr provides selective suppression of individual APs within trains of high frequency firing with high precision.

\section{Results}

The strategy to reduce blue light-mediated excitation by red-shifted channelrhodopsins

The channelrhodopsin Chrimson and its variants have the most red-shifted spectra (Klapoetke et al., 2014), and their response to $470 \mathrm{~nm}$ has a slower on-rate compared to the $590 \mathrm{~nm}$ light (Supplementary Fig. 1A-B). Despite this, $470 \mathrm{~nm}$ light is equally effective in inducing APs (Supplementary Fig. 1C). To overcome

${ }^{1}$ DANDRITE, The Danish Research Institute of Translational Neuroscience, ${ }^{2}$ Department of Molecular Biology and Genetics, Aarhus University, Denmark, ${ }^{3}$ Center for Proteins in Memory - PROMEMO, Danish National Research Foundation, ${ }^{4}$ Tasmanian School of Medicine, College of Health and Medicine, University of Tasmania, Tasmania, TAS 7000, Australia, ${ }^{5}$ Instituto de Fisiología Biología Molecular y Neurociencias (IFIBYNE), Universidad de Buenos Aires, CONICET, Buenos Aires, Argentina, ${ }^{6}$ These authors contributed equally: John Y. Lin, Sadegh Nabavi ${ }^{\dagger}$ Present address : Novo Nordisk Foundation Center for Basic Metabolic Research, Copenhagen University, Copenhagen, Denmark and Institute for Neuroscience, Copenhagen University, Copenhagen, Denmark *Correspondence : JY Lin (john.lin@utas.edu.au) and S Nabavi (snabavi@dandrite.au.dk) 
A
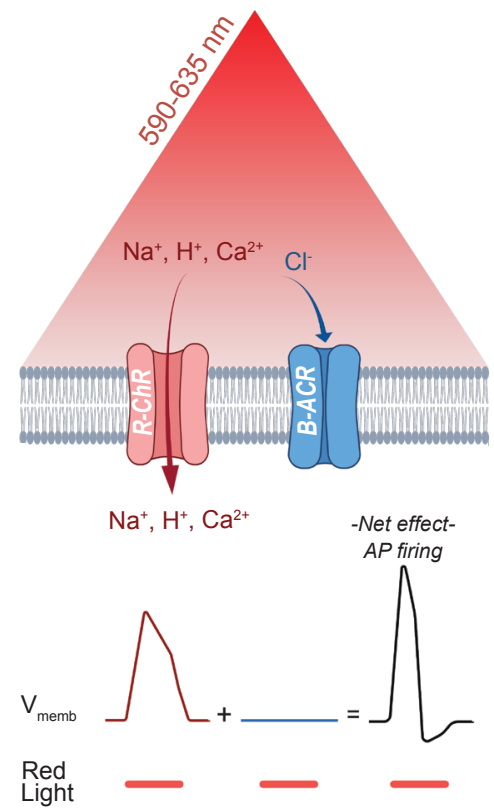

B1

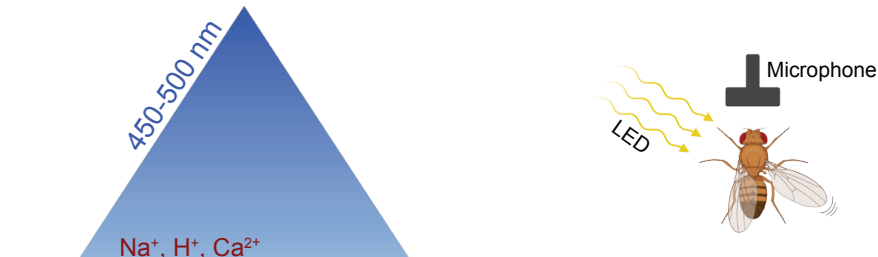

B3

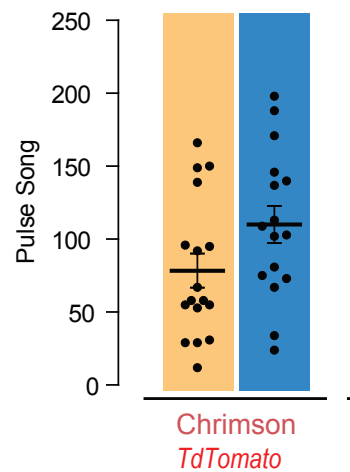

B2
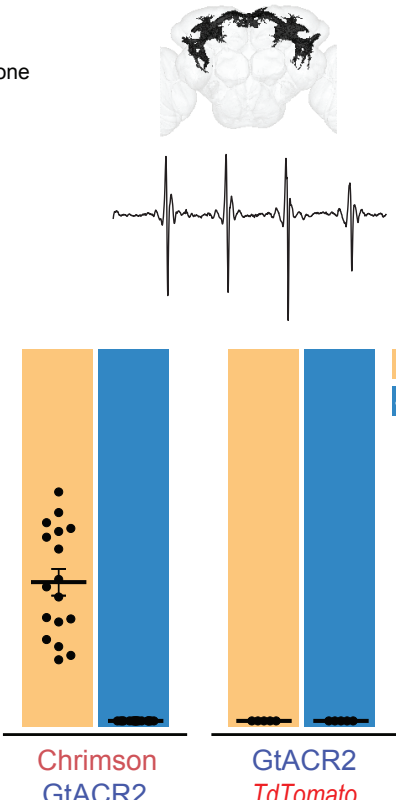

Figure 1. Reducing blue-light mediated excitation by red-shifted channelrhodopsins- Proof of concept. (A) Schematic representation of the approach. A red-shifted channelrhodopsin (R-ChR) is co-expressed with a blue-shifted anion channel (B-ACR). When red light is ON, there is an overall excitation of the cell due to the more dominant R-ChR response. When blue-light is ON, the shunting inhibitory effect of B-ACR reduces the excitation induced by R-ChR to blue light. (B) Validation of the approach in flies expressing Chrimson, Chrimson and GtACR2, or GtACR2 alone . (B1) Experimental setup to record the courtship song of solitary male flies. (B2) Top, Example of a reconstructed neuronal arborization of P1 neurons, Bottom, example of a courtship song induced by LED pulses. (B3) Courtship song production of the solitary male flies during 10-sec of constant illumination with $590 \mathrm{~nm}$ (amber) or 470 $\mathrm{nm}$ (blue) light. Each dot represents an individual (mean \pm SEM)

this limitation, we decided to take advantage of blue light-gated inhibitory chloride channels which effectively block action potential upon exposure to blue light (Govorunova et al., 2015; Govorunova et al., 2017). By co-expressing a B-ACR along with a Chrimson variant, photostimulation of targeted neurons by red light only activates R-ChR, which causes photoactivation of these neurons. Blue light, however, activates R-ChR as well as B-ACR. This allows the chloride channel to induce shunting inhibition of the neurons which in turn prevents firing of the neurons by blue light (Fig. 1A).

\section{Validation of the co-expression strategy in Drosophila}

As the first step for validating this approach, we took advantage of the well-studied male-specific P1 neurons in the fly central brain that elicit courtship singing upon activation (von Philipsborn et al., 2011; Inagaki et al., 2014; Ellendersen and von Philipsborn 2017; Mohammad et al., 2017) (Fig. 1B1-B2). When Chrimson was co-expressed with an inactive control protein (tdTomato), illumination at $470 \mathrm{~nm}$ and $590 \mathrm{~nm}$ wavelengths led to similar amounts of courtship song (respectively $110.1 \pm 12.8$ and $78.5 \pm 11.6, \mathrm{n}=16-17)$, demonstrating that blue light at the chosen intensity was efficiently activating Chrimson (Fig 1B3). When Chrimson was co-expressed with the blue-sensitive anion channelrhodopsin GtACR2, $590 \mathrm{~nm}$ light illumination reliably elicited male courtship song $(95.2 \pm 9.14)$, however, $470 \mathrm{~nm}$ light illumination did not evoke any detectable pulse song ( $\mathrm{n}=17$ 18). Neither $590 \mathrm{~nm}$ nor $470 \mathrm{~nm}$ light pulses induced courtship song in flies expressing only GtACR2 $(\mathrm{n}=5)$.

\section{Identifying a suitable pair of ultrafast light-gated anion channel and a red-shifted Chrimson variant}

Although GtACR2 proved to be effective in protocols with pulses of long duration, its slow current decay (Fig. 2A, see GtACR2, $161 \pm 24 \mathrm{~ms}, \mathrm{n}=3$ ) limits its application to optical stimulations of lower frequencies (Govorunova et al., 2015; Govorunova et al., 2017). For this reason, we searched for a pair of blue-shifted anion channelrhodopsin and red-shifted cation channelrhodopsin with fast and matching kinetics.

For a fast R-ChR, we evaluated the recently published vfChrimson, the fastest red-shifted channelrhodopsin available at this time, which can induce APs up to the intrinsic limit of the neurons (Mager et al., 2018). Previously, we found that Chrimson and its variants traffic poorly to the membrane and developed a strategy to improve the trafficking (C-Chrimson-ts, Buonaventura et al., 2019). We adapted this modification to improve the trafficking of vfChrimson (referred as IvfChrimson). In our measurements in HEK293 cells, IvfChrimson has a channel off-rate consistent with previously reported (Mager et al., 2018) (off-rate time constant of $5.6 \pm 0.3 \mathrm{~ms}, \mathrm{n}=10$ compared to C-Chrimson-ts $21.5 \pm 1.2, \mathrm{n}=$ 10 ; mean \pm SEM, Fig. 2A, Supplementary Fig. 2). When comparing C-Chrimson-ts and IvfChrimson photocurrents, we observe 1.68 fold reduction of normalized photocurrent of IvfChrimson $(0.086 \pm 0.055 \mathrm{pA} / \mathrm{pF} / \mathrm{A} . \mathrm{U}, \mathrm{n}=25)$ compared to C-Chrimson-ts 
A
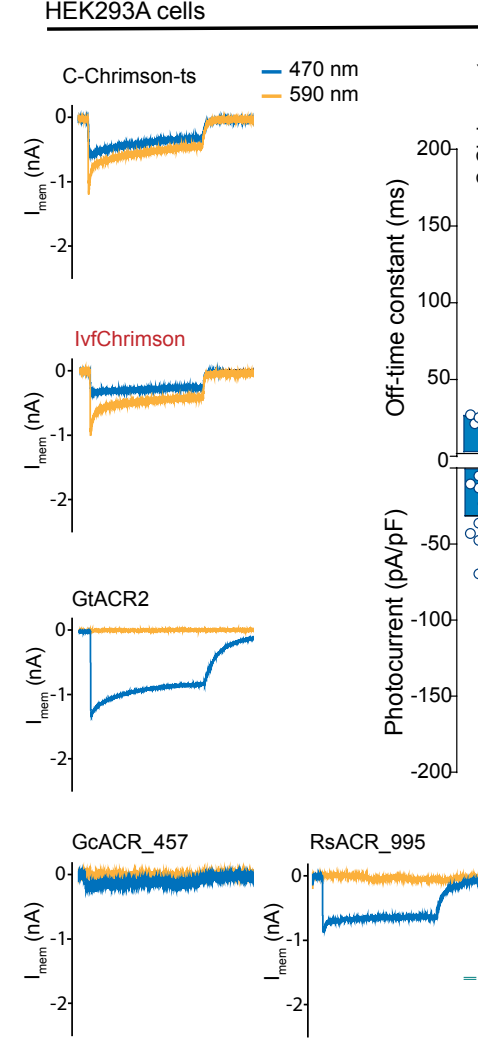
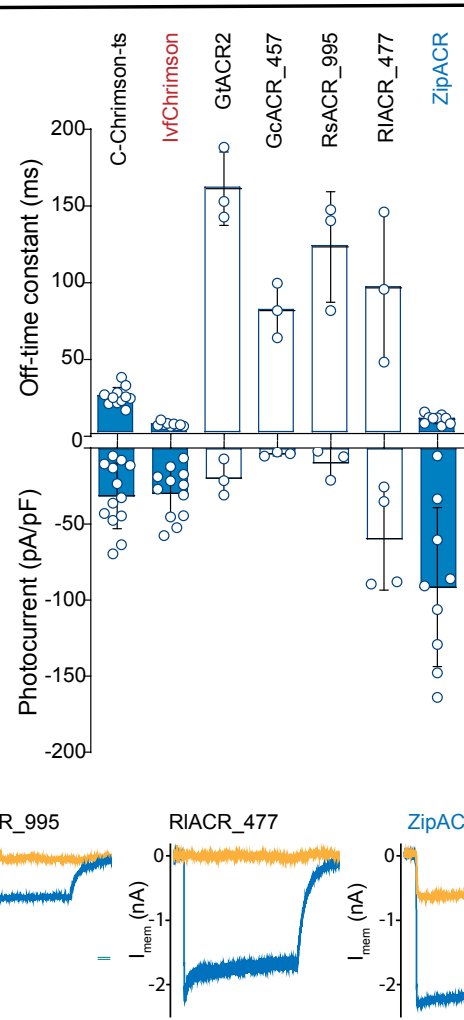

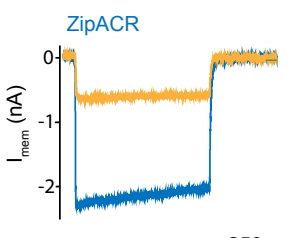

$2 \underline{50} \mathrm{~ms}$

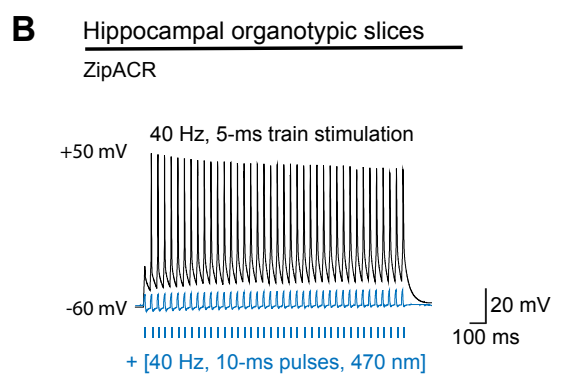

C Hippocampal acute slices

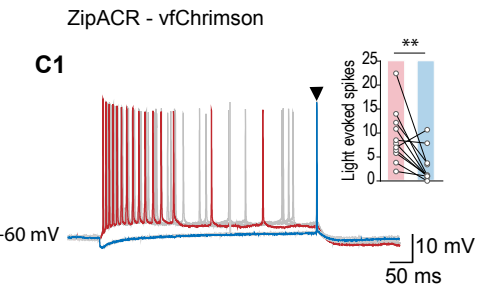

C2

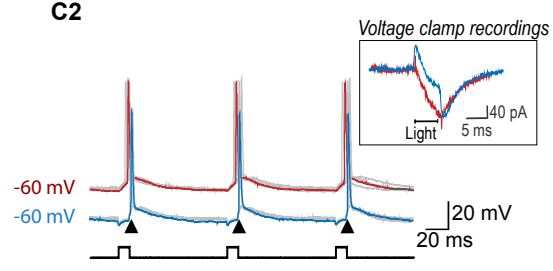

Figure 2. Comparison of channel properties of light-gated chloride channels and Chrimson variants. (A) Representative photocurrents of the Chrimson and ACRs variants in whole-cell patch-clamp recordings from HEK293 cells with $590 \mathrm{~nm}$ and $470 \mathrm{~nm}$ illumination $\left(10 \mathrm{~mW} / \mathrm{mm}^{2}\right)$. For Chrimson variants, a re-conditioning pulse at $405 \mathrm{~nm}$ illumination was applied 15 seconds before $470 \mathrm{~nm}$ LED stimulation. The plot shows the off- time constant (top) following 1 sec of $470 \mathrm{~nm}$ illumination, and the peak photocurrent (bottom) at $-60 \mathrm{mV}$ (mean $\pm \mathrm{SD}, \mathrm{n}=3-13$ cells). (B) Typical response of CA1 cells from hippocampal organotypic slices expressing ZipACR to $40 \mathrm{~Hz}, 5 \mathrm{~ms}$ pulses (top black trace) and to an illumination protocol using $40 \mathrm{~Hz}$ of $10 \mathrm{~ms}$ light pulses at $470 \mathrm{~nm}$ (bottom blue trace). (C) Recordings from dentate gyrus granule cells in acute slices expressing ZipACR and vfChrimson. (C1) Representative responses to $500 \mathrm{~ms}$ illumination at $470 \mathrm{~nm}$ and $635 \mathrm{~nm}$ (blue and red traces respectively). Note, $635 \mathrm{~nm}$ illumination evoked action potential firing while 470 $\mathrm{nm}$ illumination only evoked a single action potential time-locked to the end of the light pulse (arrowhead). Insert, plot showing the number of light-evoked spikes at $635 \mathrm{~nm}$ (red bar) and $470 \mathrm{~nm}$ (blue bar) illumination. $\mathrm{n}=11$ cells, paired t-test ${ }^{* *} \mathrm{p}<0.01$; (C2) Example of light-induced firings by $635 \mathrm{~nm}$ and 470 $\mathrm{nm}$ illumination (red and blue traces respectively, $5 \mathrm{~ms}$ pulses at $10 \mathrm{~Hz}$ ). The arrowheads show consistent APs time-locked to the end of the blue light pulse. Insert, example of a voltage-clamp recording of currents evoked by $5 \mathrm{~ms}$ pulses of $635 \mathrm{~nm}$ and $470 \mathrm{~nm}$ light (red and blue traces respectively). Light-induced currents with $470 \mathrm{~nm}$ illumination were initially outward but turned inward immediately following the light offset. Note the late inward current matches the red light induced current, reflecting a slower kinetics for vfChrimson compare to ZipACR.

$(0.145 \pm 0.126$ pA/pF/A.U., $n=25 ;$ mean \pm SD, Supplementary Fig. 2B) when the peak photocurrent amplitude was adjusted to membrane capacitance and membrane fluorescence.

To counteract the IvfChrimson excitation, we estimated that desired properties of the blue light activated anion ChR should have the off-rate time constant of $-15-30 \mathrm{~ms}$, minimal response to light of wavelength $>590 \mathrm{~nm}$ and a strong photocurrent. We identified ZipACR, RsACR_995, GcACR_457, RIACR_477 and GtACR2 as possible templates for further development based on published data (Govorunova et al., 2017). GtACR2, RsACR_995 and RIACR_477 are spectrally ideal with negligible response to $590 \mathrm{~nm}$ light (Supplementary Fig. 3A), but their kinetics are slow (mean off-rate of $161.0 \pm 24.1,122.7 \pm 36.3$ and $95.8 \pm 49.4$ ms, respectively, $\mathrm{n}=3$, Fig. 2A) which could have undesired long lasting inhibitory effects after light termination. GcACR_457 has very small photocurrents and cannot be characterized accurately, possibly due to poor membrane trafficking. ZipACR, although spectrally not ideal ( $-515 \mathrm{~nm}$; Govorunova et al., 2017) has a kinetic close to the desired range $(9.5 \pm 3.0 \mathrm{~ms}, \mathrm{n}=9)$ and it has strong photocurrents (Fig. 2A).

To test the inhibitory property of ZipACR, we injected $40 \mathrm{~Hz}$ somatic current in organotypic hippocampal neurons expressing the channel. Consistent with the original report, individual pulses of $470 \mathrm{~nm}$ light resulted in the time-locked suppression of APs (Fig. 2B).

Next we examined whether ZipACR can block blue light-mediated APs in neurons expressing vfChrimson. Recordings from the granule cells in acute hippocampal slices co-expressing ZipACR and vfChrimson showed that, compared with $635 \mathrm{~nm}$ light, 470 $\mathrm{nm}$ light induced significantly fewer APs (Fig. 2C1), in line with our prediction. However, we consistently observed the appearance of individual APs time-locked to the blue light offset (Fig. 2C1$\mathrm{C} 2$ ). We concluded that due to the fast closing rate, the photoinhibitory current generated by ZipACR decays too 
A1

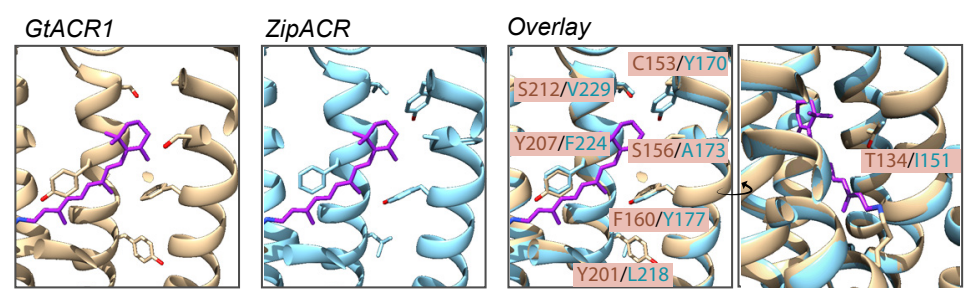

A2

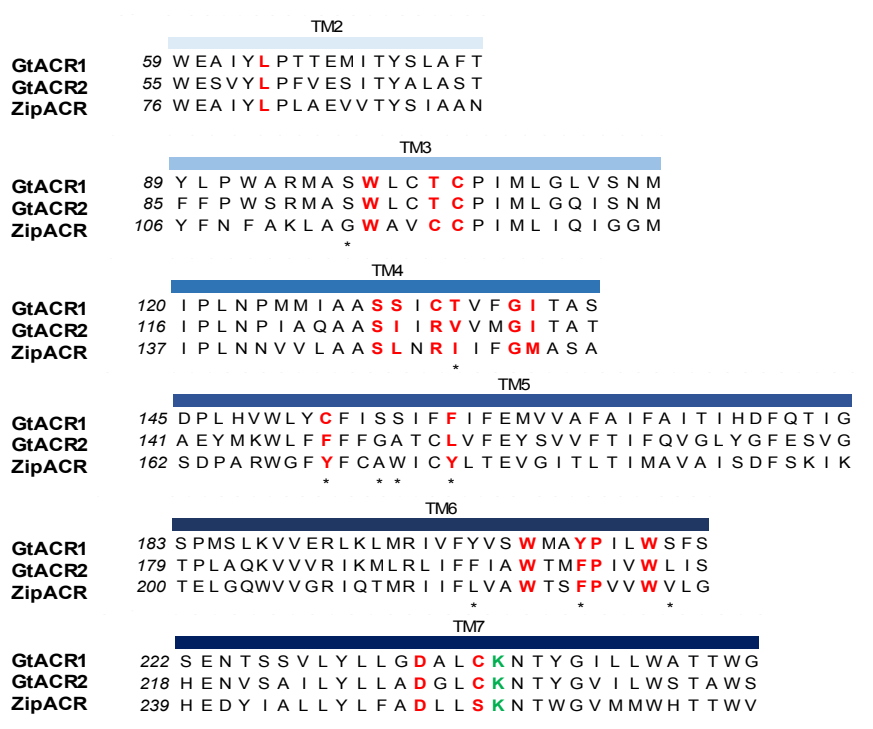

B

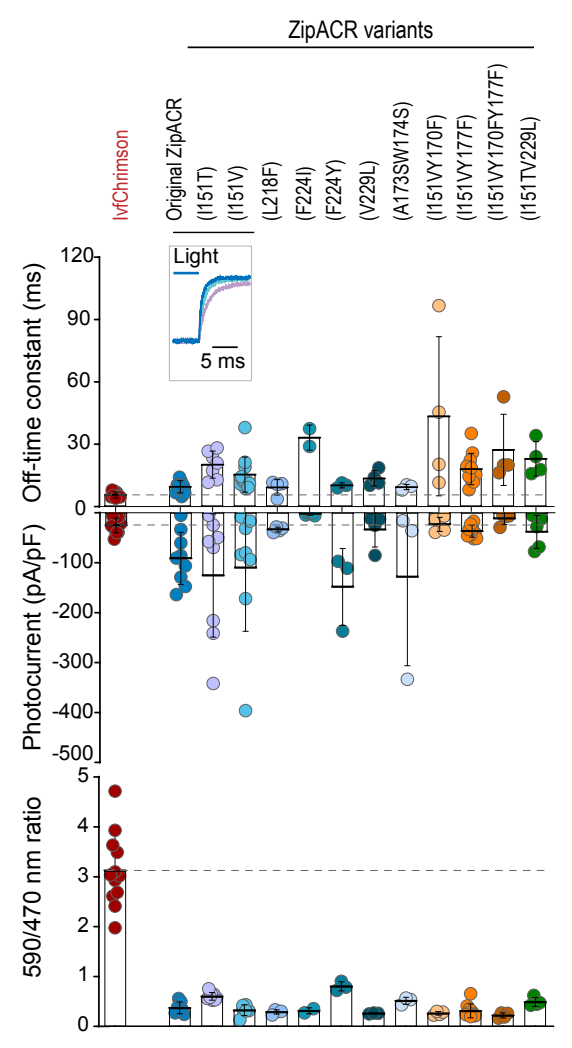

Figure 3. Characterization of the optimized variants of the ultrafast anion channelrhodopsin ZipACR. (A) Structure-based design of ZipACR variants. (A1) The retinal binding pocket of GtACR1 and the putative retinal binding pocket of ZipACR homology model. (A2) The alignment of the transmembrane domains (TM) of GtACR1, GtACR2 and ZipACR. The residues surrounding the retinal binding pocket of GtACR1 and the corresponding residues from GtACR2 and ZipACR are highlighted in red. *marks the residues targeted for mutation. The lysine that forms the Schiff base with retinal is indicated in green. The numbers on the left correspond to the number of the first residue in each line. (B) Basic properties of the ZipACR variants compared to IvfChrimson. off-time constant (top) following $1 \mathrm{sec}$ of $470 \mathrm{~nm}$ illumination, peak photocurrent (middle) and 590/470 ratio (bottom). The data have been obtained from whole-cell recordings in HEK293 cells. Each dot corresponds to one cell (mean \pm SD, $\mathrm{n}=2-12$ ).

early to suppress the remaining excitatory response of vfChrimson.

Therefore, we decided to generate variants of ZipACR with slightly slower off-kinetics.

\section{Optimizing ZipACR off-kinetics}

It is technically easier to generate mutations with slower kinetics and blue-shifted spectra than generating variants with faster onand off-kinetics. Thus, we decided to use ZipACR as a template for further mutagenesis instead of further modifying GtACR2. To identify relevant mutation sites in ZipACR, we generated a homology model of ZipACR with the crystal structure of GtACR1 (Govorunova et al., 2017) and identified the putative residues forming the retinal binding pocket in ZipACR (Fig. 3A). Multiple alignments were performed with the kinetically slower GtACR1 and GtACR2 to identify possible replacement of these residues that would result in slower kinetics and/or small blue-shift in ZipACR without disrupting the channel function (Fig. 3A2). We tested these variants in HEK293 cells for photocurrent ratio 590 $\mathrm{nm} / 470 \mathrm{~nm}$ LED stimulating light and measured the channel off-rate kinetics and photocurrent amplitude (Fig. 3B). Of these candidates, we identified I151V and I151T at the 4th transmem- brane domain of ZipACR that resulted in a small increase in channel off-rate time constant $(15.5 \pm 8.4 \mathrm{~ms}$ for I151V and 20.2 $\pm 6.4 \mathrm{~ms}$ for I151T, mean \pm SD, Fig. 3B) compared to the original ZipACR $(9.5 \pm 2.9 \mathrm{~ms}$, mean $\pm \mathrm{SD})$. We also generated double and triple mutants combining the I151V or I151T with Y170F, Y177F or V229L mutations but most of these double or triple mutants appeared to have smaller photocurrents and off-rate kinetics that could not be fitted well with single exponential back to zero baseline. Based on these results, we concluded the variants I151V (Zip(151V)) and I151T (Zip(151T)) are the best candidates for the pairing and proceeded for further characterizations.

Neither Zip(151V) nor Zip(151T) mutations changed the ion permeability of ZipACR as measured by channel reversal potential in potassium gluconate and cesium chloride based intracellular recording solutions (Supplementary Fig. 3B-C). In terms of light sensitivity, it is desirable that the ZipACR variant would respond to $470 \mathrm{~nm}$ light faster and stronger and to red-shifted light slower and weaker than IvfChrimson at the same intensity (Supplementary Fig. 4). With both Zip(151V) and Zip(151T), the channel on-rate kinetics have the desirable 
C1

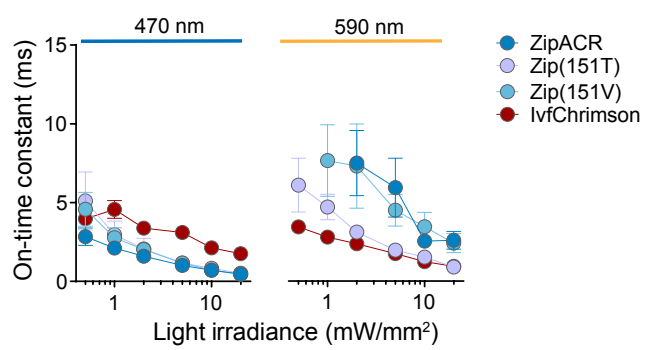

D1

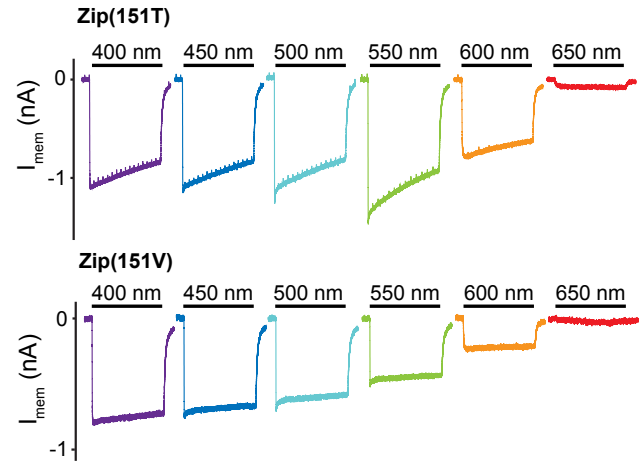

E1

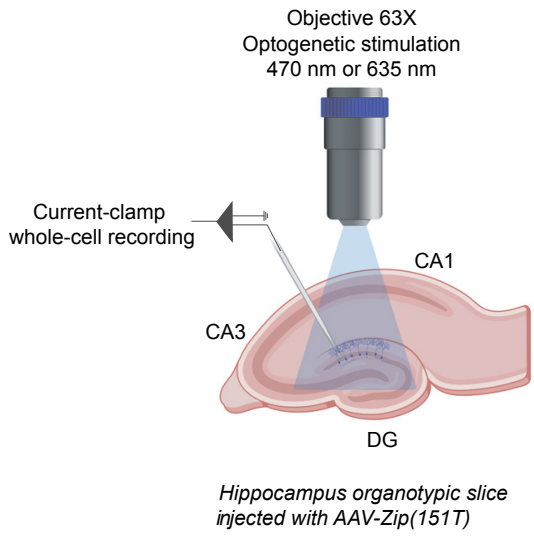

C2

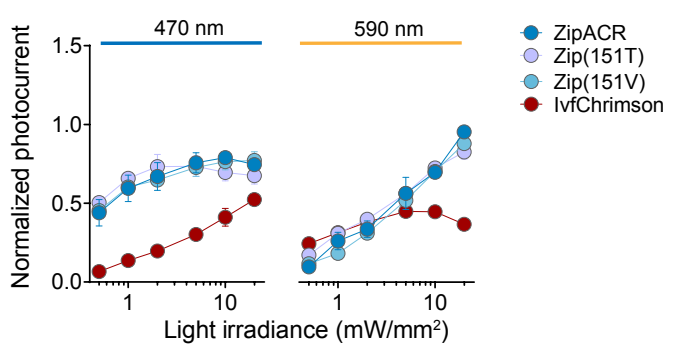

D2

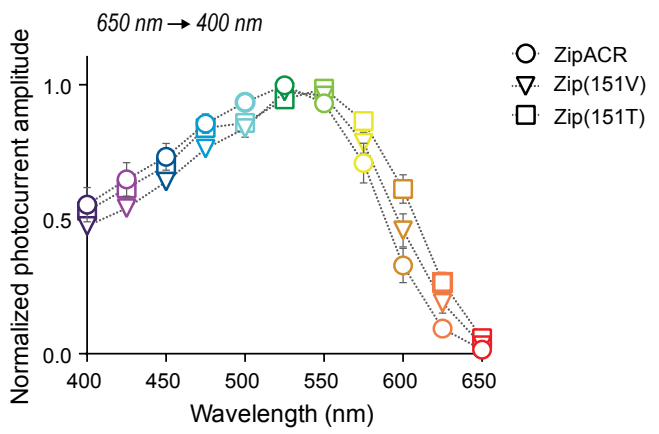

E2

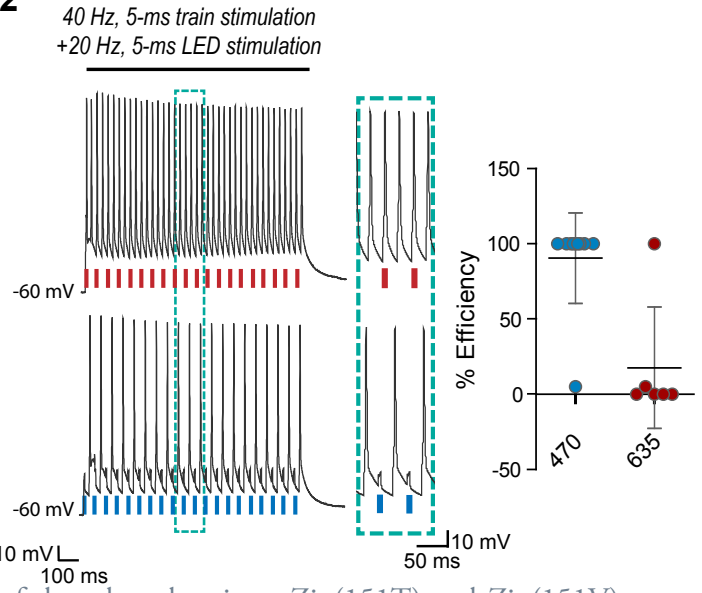

Figure 3 (C) On-time constant (C1) and normalized photocurrent (C2) of the selected variants Zip(151T) and Zip(151V) as compared to the original ZipACR and IvfChrimson, with $470 \mathrm{~nm}$ or $635 \mathrm{~nm}$ illumination of various light intensities (in $\mathrm{mW} / \mathrm{mm}^{2}$ ). For the photocurrent, the values have been measured at steady-state. The data points are the mean value \pm SEM $(n=6-7)$. (D) The responses of the selected variants to various wavelengths of the same photon flux. (D1) The action spectra of ZipACR, Zip(151V) and Zip(151T) as measured from $650 \mathrm{~nm}$ to $400 \mathrm{~nm}$ (D2) For the action spectra, the maximum photocurrent amplitudes measured at each wavelength were normalized to the peak values obtained from the same cell across the spectrum. The data points are the mean value \pm SEM $(n=6-7)$. (E) Validation of the efficiency of the Zip(151T) variant in slices. (E1) The response of dentate gyrus granule cells expressing $\mathrm{Zip}(151 \mathrm{~T}$ ) variant at $470 \mathrm{~nm}$ and $635 \mathrm{~nm}$ light pulses delivered through a 63x objective. (E2) Representative traces of the firing induced by current injection at $40 \mathrm{~Hz}$. Overlapping $470 \mathrm{~nm}$ but not $635 \mathrm{~nm}$ pulses block action potentials. The plot on the right shows the efficiency (in \%) of $\mathrm{Zip}(151 \mathrm{~T})$ in blocking individual action potentials at 470 and $635 \mathrm{~nm}$ light pulses (mean $\pm \mathrm{SD}, \mathrm{n}=6-10$ cells). The traces of the two outliers are presented in supplementary Fig. 5A.

properties as described above to $470 \mathrm{~nm}$ and $590 \mathrm{~nm}$ light, although the $\mathrm{Zip}(151 \mathrm{~T})$ on-rate starts to approach the on-rate of IvfChrimson at light intensities $>5 \mathrm{~mW} / \mathrm{mm}^{2}$ at $590 \mathrm{~nm}$ (Fig. 3C1). With $470 \mathrm{~nm}$ light, IvfChrimson does not appear to reach saturating response even at $20 \mathrm{~mW} / \mathrm{mm}^{2}$ (Fig. 3C2). With 590 $\mathrm{nm}$ light, IvfChrimson reaches saturating response at $10 \mathrm{~mW} /$ $\mathrm{mm}^{2}$ whereas none of the ZipACR variants appears to reach saturating response even at $20 \mathrm{~mW} / \mathrm{mm}^{2}$ (Fig. 3C2). Spectrally, Zip(151T) shows a wider red-shifted action spectra than ZipACR whereas Zip(151V) may have a slightly narrower blue-shifted ac- tion spectra than ZipACR (Fig. 3D).

Since $\mathrm{Zip}(151 \mathrm{~T})$ is a more red-shifted variant of the two (Fig. 3B,D), we asked whether red light pulses may induce photoinhibitory currents that suppress APs. We expressed Zip(151T) in the granule cells of the dentate gyrus in hippocampal slices using AAV vector and tested its inhibitory effect upon $470 \mathrm{~nm}$ and 635 $\mathrm{nm}$ light pulses. We injected somatic current at $40 \mathrm{~Hz}$, while alternately delivering overlapping $5 \mathrm{~ms}$ pulses of light. Except in two instances, blue light pulses were consistently effective in blocking APs, while red light had no effect on APs induc- 
bioRxiv preprint doi: https://doi.org/10.1101/2021.05.05.442824; this version posted May 6, 2021. The copyright holder for this preprint (which was not certified by peer review) is the author/funder. All rights reserved. No reuse allowed without permission.

A
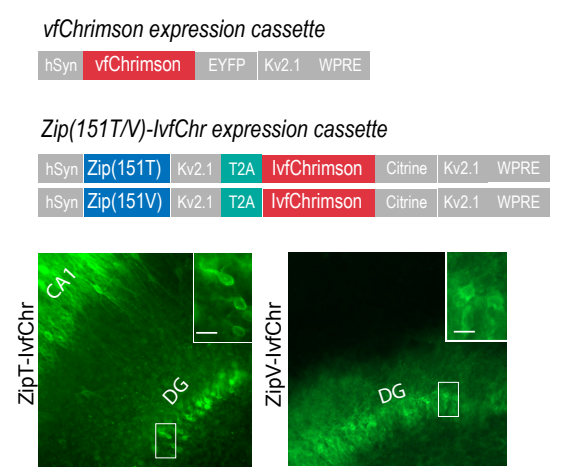

C1

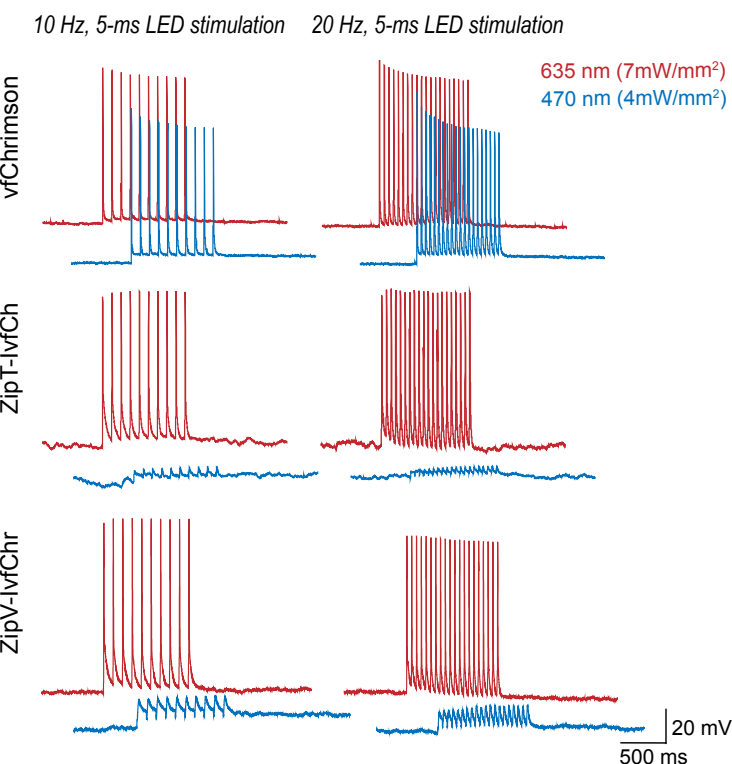

C2

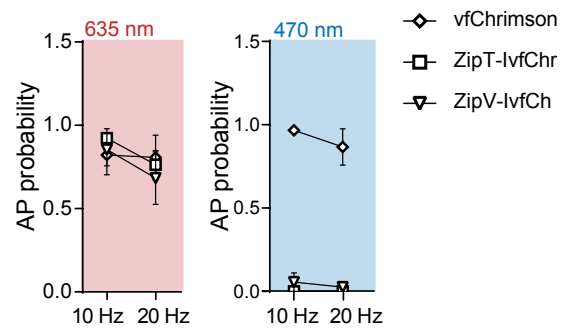

B

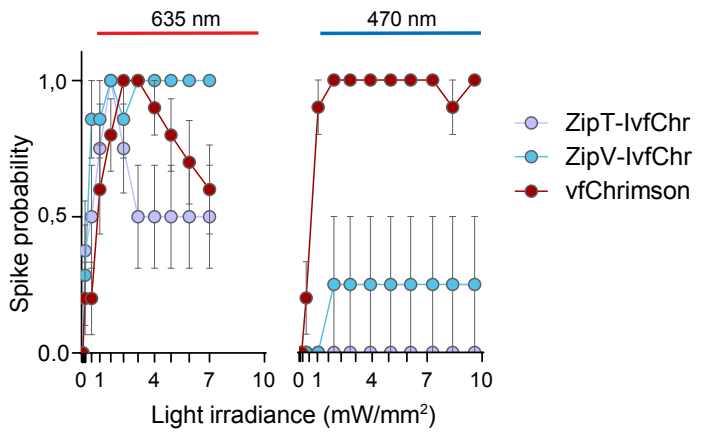

D

$40 \mathrm{~Hz}, 5-m s$ train stimulation $+20 \mathrm{~Hz}, 5-m s$ LED stimulation
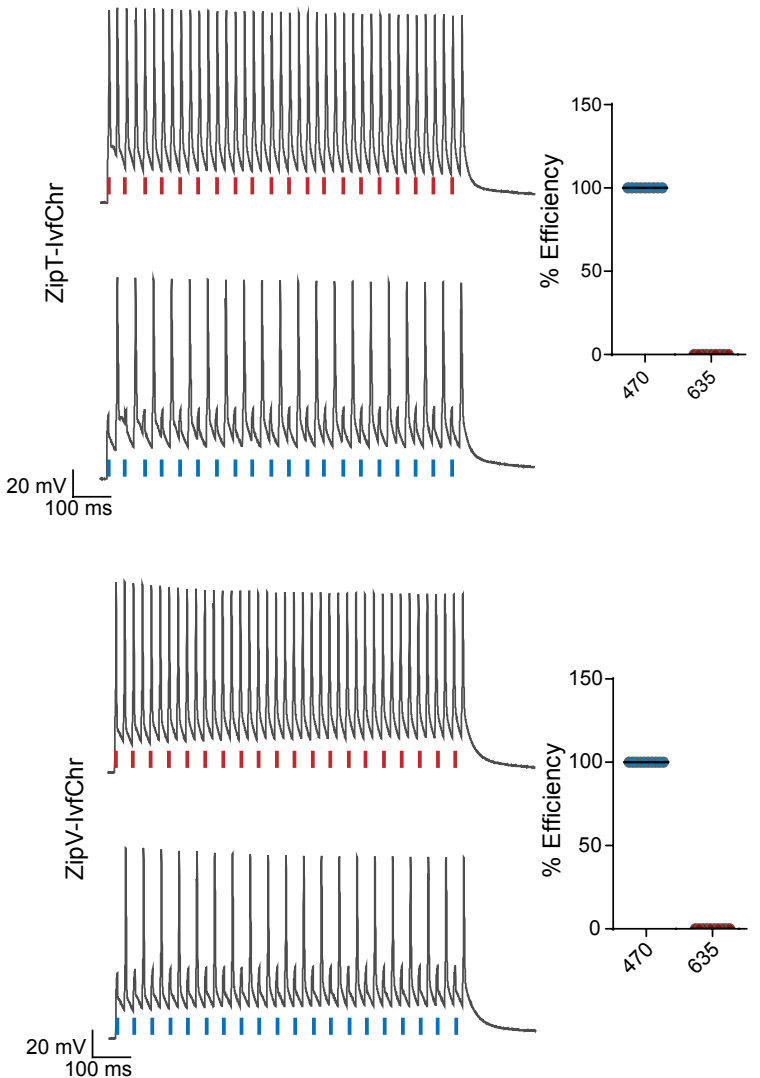

Figure 4. ZipACR variants co-expressed with the red ChR2 IvfChrimson prevent blue-light induced action potentials. (A) The expression cassette for vfChrimson and bicistronic Zip-IvfChr variants used in rAAV vector (Top). The selected ZipACR variants (Zip(151T) or Zip(151V)) are co-expressed with the red-sensitive IvfChrimson by using the 2A self-cleaving peptide (T2A). All the opsins are soma-targeted with a Kv2.1 peptide. (Bottom) Representatives showing the membrane enriched expression of ZipT-IvfChr and ZipV-IvfChr. Scale bar $=25 \mathrm{um}$. (B) Blue and red light- driven spike fidelity at various light intensities in DG cells ( $\mathrm{n}=4-10$ cells for each opsin; $10 \mathrm{~ms}$ pulse width, single pulse). (C) Comparison of the high-frequency red and blue light-induced spiking (C1), and spike probability (C2, n = 6-13 for each opsin), of DG cells expressing vfChrimson or the Zip-IvfChr variants, with 10 and $20 \mathrm{~Hz}$ of light pulses $\left(4-7 \mathrm{~mW} / \mathrm{mm}^{2}\right)$. Holding potential $=-60 \mathrm{mV}$. (D) Optical inhibition of spiking in DG cells expressing the Zip-IvfChr variants. Representative traces of the firing induced by current injection at $40 \mathrm{~Hz}$. Overlapping $470 \mathrm{~nm}$ but not $635 \mathrm{~nm}$ light pulses block action potentials. Holding potential = $-60 \mathrm{mV}$. The plots on the right show the efficiency (in \%) of ZipT-IvfChr and ZipV-IvfChr in blocking individual action potentials at 470 and $635 \mathrm{~nm}$ ( $\mathrm{n}$ $=9-10$ for each opsin).

tion (Fig. 3E, $\mathrm{n}=10$ for 470 and $\mathrm{n}=6$ for 635 , the two outliers are presented in Supplementary Fig. 5A). It has been reported that at resting or hyperpolarized membrane potentials ZipACR may produce light-evoked APs (Kato et al., 2018). We did not observe such a phenomenon under our experimental setup (Supplementary Fig. 5B).

\section{Validation of the co-expression strategy in brain slice}

Since co-injection of two viruses results in variability in the ratio of expression in different neurons, we created constructs containing IvfChrimson and Zip(151T/V) (ZipT-IvfChr and ZipV-IvfChr) using the bicistronic $2 \mathrm{~A}$ sequence (Fig. $4 \mathrm{~A}$ ). This ensures 
an equimolar expression of the two channels. We also introduced a soma targeting Kv2.1 sequence into the constructs while eliminating the endoplasmic reticulum-exporting sequence of IvfChrimson construct. Based on the photocurrent amplitudes of the constructs in response to $590 \mathrm{~nm}$ light (at $-40 \mathrm{mV}$ holding potential with potassium gluconate-based intracellular solution to minimize Zip(151V) variant responses), the photocurrent amplitudes of IvfChrimson-only and ZipV-IvfChr constructs are comparable after adjustment to cell size and membrane fluorescence in HEK293 cells (Supplementary Fig. 6). We did not test the ZipT-IvfChr variant as the red-shifted response of $\mathrm{Zip}(151 \mathrm{~T})$ is difficult to eliminate to $590 \mathrm{~nm}$ light.

As the first step to test the performance of the system, we examined the power at which $470 \mathrm{~nm}$ and $635 \mathrm{~nm}$ lights induce APs in ZipT-IvfChr and ZipV-IvfChr, as well as in vfChrimson expressing neurons. We expressed AAV constructs in the granule cells of the dentate gyrus in the hippocampal slices (as described in Fig. 3E1). We recorded cells under the current-clamp mode and injected current through the patch pipette to maintain the membrane potentials at $-60 \mathrm{mV}$. As expected, in vfChrimson expressing neurons $470 \mathrm{~nm}$ and $635 \mathrm{~nm}$ lights induced APs with light powers as low as $0,5 \mathrm{~mW} / \mathrm{mm}^{2}(\mathrm{n}=10$, Fig. $4 \mathrm{~B})$. In contrast, ZipT-IvfChr expressing neurons produced APs only to 635 $\mathrm{nm}$ light stimulation, whereas $470 \mathrm{~nm}$ light powers as high as 10 $\mathrm{mW} / \mathrm{mm}^{2}$ failed to induce APs ( $\left.\mathrm{n}=7-8\right)$. As for ZipV-IvfChr, we occasionally observed blue light-induced APs ( $n=4-7$, Fig. 4B), which may be explained by its relatively faster decay time (Fig. $3 \mathrm{~B})$. In neurons expressing vfChrimson, we noticed a reduction in probability of AP upon successive optical stimulation specifically to $635 \mathrm{~nm}$ pulses (Fig. 4B). Consistent with this, we observed a reduction in response in HEK293 cells expressing IvfChrimson (Supplementary Fig. 7A,B). This phenomenon, likely caused by the desensitization of the channel, is consistent with previous reports (Lin et al., 2013).

To examine the efficiency of $635 \mathrm{~nm}$ and $470 \mathrm{~nm}$ lights in inducing high frequency APs, we delivered $10 \mathrm{~Hz}$ and $20 \mathrm{~Hz}$ light pulses of $5 \mathrm{~ms}$ duration (Fig. 4C). As expected, in vfChrimson expressing neurons both $470 \mathrm{~nm}$ and $635 \mathrm{~nm}$ lights induced APs (n = 8-9). In neurons expressing either ZipT-IvfChr or ZipV-IvfChr, $635 \mathrm{~nm}$ pulses were equally effective in driving APs. On the other hand, $470 \mathrm{~nm}$ light only induced modest depolarization, which never reached the threshold for AP induction ( $\mathrm{n}=5-12$ for each opsin) (Fig. 4C2).

To test the temporal precision of the system in blocking AP, we stimulated the neurons by somatic current injection at $40 \mathrm{~Hz}$, while alternately delivering perfectly overlapping $5 \mathrm{~ms}$ pulses of lights. Light pulses of $470 \mathrm{~nm}$ were consistently effective in blocking APs, while $635 \mathrm{~nm}$ light pulses had virtually no effect on APs induction as its amplitude, half-width and delayed time were indistinguishable from APs induced in the absence of light pulse (Fig. 4D).

Local mono-synaptic connection is a common feature in many brain regions. For this reason, independent dual optical activation requires that while optically stimulating pre-synaptic neurons, post-synaptic neurons remain responsive to the pre-synaptic inputs. To test whether our system meets this criterion, we asked how fast a neuron recovers from the inhibition induced by the ZipACR variants. We induced APs through somatic current injection at different time points from the offset of a light pulse. Blue light pulses blocked APs if the current was injected simultaneously. As expected from the off-rate of $\operatorname{Zip}(151 \mathrm{~T})$, within $5 \mathrm{~ms}$ from the light offset neurons expressing Zip(151T) or ZipT-IvfChr produced APs with high probability (Supplementary Fig. 8 ). The success rate reached its maximum within 10-15 ms. The recovery time for ZipV-IvfChr was immediate, with 100\% success rate immediately following the light offset. This indicates that ZipV-IvfChr could be used for dual optical activation without off target inhibition.

\section{Discussion}

The main purpose of this work was to find a practical solution to the problem of cross-over where all opsins, regardless of their preferred excitation spectra, are activated by blue light. We describe a R-ChR2 expression system by which pulses of red light drive time-locked high frequency APs, while blue light remains ineffective. We created this system by pairing a B-ACR with the ultrafast red-shifted ChR2, IvfChrimson. This requires the B-ACR to have a significantly larger photocurrent compared to R-ChR2 as well as a faster channel opening and slightly slower channel closing. For this reason, we chose ZipACR as the template B-ACR for further modifications. Based on our homology modelling and multiple alignments with the kinetically slower B-ACRs, GtACR1 and GTACR2, we identified potential residue replacements within the putative retinal binding pocket which would result in slightly slower kinetics without impairing the channel function.

To our surprise, many mutations of ZipACR around all-trans retinal do not alter the channel properties or compromise channel conductance too strongly. This is contrary to our past experiences with channelrhodopsins where conductance is highly sensitive to mutations around the retinal binding pocket (data not shown). ZipACR double mutants appear to have more compromised conductance or altered properties. Among the tested positions, the I151 residue appears to have significant influences in kinetics and spectral properties. Indeed, we found that two variants, Zip(151T) and Zip(151V), sit in a sweet spot for our purpose. More specifically, the channels off-rate kinetics have the desirable property of being slightly but sufficiently slower than Ivf- 
Chrimson without compromising the opening rate, photocurrent and light spectrum. Thus, when paired with IvfChrimson, they prevent AP induction by blue light, without interfering with red light-induced high frequency neural spiking. On a similar note, although blue light pulses produced subthreshold depolarization, we did not observe charge integration that could have resulted in AP, even in frequencies as high as $20 \mathrm{~Hz}$; this most likely reflects the reverse potential of the ZipACR. Of the two variants, we observed Zip(151T), and consequently ZipT-IvfChr, to be more consistent in blocking blue light-induced APs, which could be explained by its slightly slower closing rate. Although Zip(151T) is the more red-shifted of the two, within the tested range of light intensities and expression, only rarely did we observe a block of APs at $635 \mathrm{~nm}$. However, we do not recommend the use of orange light pulses, as we observed a significant photocurrent in this wavelength.

Based on its impact on the kinetics and spectral properties, it will be of value to conduct saturating mutagenesis study on I151 residue that may produce variants of interesting properties or pairing that could outperform Zip(151V) and Zip(151T).

A recent study (Vierock et al., 2020) proposed a R-ChR2 expression system by relying on the pairing between GtACR2 and Chrimson, as we did in our early stage work with Drosophila. For physiological as well as experimental reasons, the priority we set was to create a system with fast closing kinetics. As channelrhodopsins are known to conduct calcium ions, long opening lifetime can evoke significant elevation of intracellular calcium (Lin et al., 2009) and activate second messenger pathways with complicating biological effects in experiments of long duration. The same concern applies to B-ACRs with slow closing kinetics as they could have additional inhibitory effects after the termination of blue light and alter the physiology to be studied. From an experimental viewpoint, a fast photostimulation system has the flexibility to perform a wide range of optical manipulations. This includes driving neuronal activity to their firing limits as well as suppressing their firing with single spike precision. Although not tested here, our results indicate that the fast closing kinetics of the B-ACR channels, particularly ZipV-IvfChr, should allow dual optical activation of monosynaptically connected neurons.

Before concluding, we must note that our approach, which runs based on chloride influx is unlikely to be suitable for axonal terminal activation, where the chloride concentration is high (Mahn et al., 2016; Mahn et al., 2018). For this matter, we refer the readers to some published methods by which independent dual channel photostimulation of the axons might be feasible (Klapoetke et al., 2014; Hook et al., 2015). Thus, our system complements these studies as they are unsuitable for somatic photoactivation. This provides researchers with complementary methods to choose from based on their experimental needs.

\section{Methods}

\section{Drosophila optogenetic activation experiments}

Flies (Drosophila melanogaster) were kept on standard food medium (water, cornmeal, oatmeal, sucrose, yeast, agar, acetic acid, preservative methyl-4-hydroxybenzoate) at $25^{\circ} \mathrm{C}, 60 \%$ humidity, and a $12 \mathrm{~h} / 12 \mathrm{~h}$ light-dark cycle. Male flies were collected after eclosion and aged for 7 days in isolation and protected from light on standard food, followed by 3 days on food containing $400 \mu \mathrm{M}$ all-trans retinal (Sigma). The following genotypes were assayed: co-expression of Chrimson and GtACR2: w-; NP2631.GAL4/ UAS-CsChrimson.mVenus.attp40; tubP-FRT-stop-FRT-GAL80, fruFLP/ UAS-GtACR2.attp2; co-expression of Chrimson and tdTomato: w-; NP2631.GAL4/ UAS-CsChrimson.mVenus.attp40; tubP-FRT-stop-FRT-GAL80, fruFLP/ UAS-myr-tdTomato. attp2; co-expression of GtACR2 and tdTomato: w-; NP2631. GAL4/ UAS-myr-tdTomato.attp2; tubP-FRT-stop-FRT-GAL80, fruFLP/ UAS-GtACR2.attp2.

For optogenetic activation experiments, we used a previously described multi-channel array of electret condenser microphones (CMP-5247TF-K, CUI Inc), amplified with a custom-made circuit board and digitized with a multifunction data acquisition device (NI USB-6259 MASS Term, National Instruments) (von Philipsborn et al., 2014). Single male flies were placed in recording chambers equipped with microphones at the bottom and light diodes (Amber $591 \mathrm{~nm}$ : Cree 5-mm C503B-AAS/AAN-015 or Blue 470 nm: Cree 5-mm C503B-BAS-CY0C0461) were placed $1 \mathrm{~cm}$ above the song recording chambers at a $60^{\circ}$ angle, illuminating the whole chamber (10-sec of constant light). Tentative pulse song was detected by a MATLAB script and corrected manually, using a custom made user interface for visualization and annotation of song oscillograms (von Philipsborn et al., 2014).

\section{Cell culture, transfection and stable cell line generation}

HEK293 cells (Invitrogen) were grown in DMEM (1g/L glucose) supplemented with $8 \%$ FBS and $1 \%$ Pen/Strep. For recordings, cells were plated at low density on glass coverslips and kept in DMEM media supplemented with 4\% FBS and 1\% Pen/Strep. Transfection was done with Xtremegene 9 (Sigma-Aldrich) according to the manufacturer's instruction. Recordings of the cells were performed between 38-50 hours after transfection.

For generation of recombinant lentivirus for stable HEK293 cell lines expressing IvfChrimson and ChrimsonR-tdTomato cells, HEK293 cells were co-transfected with psPAX2 and pMD2.G (courtesy of Trono laboratory) and the media was transferred to a new dish with untreated cells 2 and 3 days after transfection. The cells receiving the media were kept as stable cell 
lines of respective constructs.

\section{Structure alignment and modelling}

Homology modelling of ZipACR structure was performed with Modeller within the UCSF Chimera to the published GtACR1 structure (PDB \#6CSM). This was further aligned to the GtACR2 alignment from Govorunova et al., (2017). Residues surrounding the retinal binding pocket of GtACR1 and the modelled ZipACR were identified and those residues that were different in ZipACR were changed to the corresponding residues of GtACR2 or GtACR1.

\section{Molecular biology}

For GcACR_457, RsACR_995 and RIACR_477, the cDNA were in the original pcDNA3.1 vector as EYFP fusion as available on Addgene (\#103137, 103772, 103771). The GtACR2 construct was synthesized (IDT DNA) and modified with a N-terminal signalling peptide from the commercial pDisplay vector (Invitrogen) and a trafficking signal prior to tdTomato or citrine fusion and inserted in the pcDNA3.1+ vector (Invitrogen) for expression. For the ZipACR constructs, the original EYFP fusion from Addgene (\#88844) was retained and the construct was either inserted into the pcDNA3.1+ vector or a second generation lentiviral vector pLenti with CMV promoter. No functional or fluorescent difference in the expressed protein were observed between expression from pcDNA3.1+ and pLenti vector in HEK293 cells and the results were combined for comparison and analysis.

Site-directed mutagenesis of the ZipACR, Chrimson and GtACR2 were performed with overlap extension PCR using Phusion DNA Polymerase (Life Technologies) and ligated into the pcDNA3.1+ or pLenti expression vector.

For ChrimsonR and vfChrimson, additional improvements for membrane trafficking was used as previously described (Buonaventura et al., 2019). In brief, N-terminal leading sequence from ChR1/oChIEF/ReaChR was transplanted to the transmembrane A-G of ChrimsonR and vfChrimson. Trafficking sequence was added after the transmembrane $\mathrm{G}$ before fusion to tdTomato. The modified ChrimsonR/vfChrimson-tdTomato constructs were inserted into the 2nd generation lentiviral pLenti vector with CMV promoter and used to make stable ChrimsonR/vfChrimson-tdTomato HEK293 cell lines. The improved vf-Chrimson is named IvfChrimson.

For the comparison of IvfChrimson-citrine and Zip(151V)Kv2.1-2A-IvfChrimson-citrine-Kv2.1 (ZipV-IvfChr) photocurrents, the two cDNAs were inserted into pcDNA3.1+ for expression. The IvfChrimson in both designs contains the modified $\mathrm{N}$-terminal sequence from ChR1/oChIEF/ReaChR but the trafficking sequence was only used in the IvfChrimson-citrine design and not the Kv2.1 design. $r A A V$ production

rAAV2-retro containing vfChrimson_EYFP_Kv2.1, Zip(151T)Kv2.1_IRES_DsRed, Zip(151T)-Kv2.1-2A-IvChrimson-citrineKv2.1 (ZipT-IvfChr), and Zip(151V)-Kv2.1-2A-IvfChrimsoncitrine-Kv2.1 (ZipV-IvfChr) were produced according to the protocol at https://www.salk.edu/science/core-facilities/viral-vector-core/resources/. In brief, HEK293A cells (Life Technologies, Carlsbad, CA) were grown to $90 \%$ confluency and transfected with vectors containing opsin proteins, and the helper plasmids XX6-80 and rAAV2-retro (gift from A. Y. Karpova, Howard Hughes Medical Institute Janelia Farm Research Campus). rAAV2-retro were released and purified according to protocol at

https://www.takarabio.com/documents/User\%20Manual/6232/6232_e.v1509Da.pdf.

\section{Electrophysiological characterization and imaging of HEK293 cells}

Whole-cell patch-clamping was performed with Axopatch 200A patch-clamp amplifier with Digidata 1322A data acquisition board and pCLAMP10 software. Cells were clamped at $-60 \mathrm{mV}$ except in experiments with the ramp protocol and experiments comparing the $590 \mathrm{~nm}$ light-induced photocurrents between IvfChrimson-citrine and ZipV-IvfChr expressing cells. In the experiment comparing the IvfChrimson-citrine photocurrent to the $2 \mathrm{~A}$ configuration, cells were clamped at $-40 \mathrm{mV}$ to reduce the influence of ZipACR response with IvfChrimson photocurrent. Series resistance for the recorded cells were mostly under $15 \mathrm{M} \Omega$ and series resistance were compensated up to $70 \%$ during recordings where possible.

The extracellular solution was composed of (in $\mathrm{mM}$ ): $145 \mathrm{NaCl}, 3$ $\mathrm{KCl}, 2 \mathrm{CaCl} 2,1 \mathrm{MgCl}$, 20 D-glucose and 10 HEPES ( $\mathrm{pH}$ 7.35). For most experiments, the intracellular solution contained (in $\mathrm{mM}$ ): $110 \mathrm{CsCl}, 15 \mathrm{TEACl}, 10$ HEPES, 5 Cs2EGTA, $1 \mathrm{MgCl} 2$ and $0.1 \mathrm{mM} \mathrm{CaCl} 2$ ( $\mathrm{pH} 7.25)$. In experiments comparing the 590 $\mathrm{nm}$ light-induced photocurrents between IvfChrimson-citrine and ZipV-IvfChr expressing cells and some ramp experiments, a K-gluconate based intracellular solution containing: $115 \mathrm{~K}$-gluconate, $10 \mathrm{~K} 2 \mathrm{EGTA}, 10 \mathrm{HEPES}, 5 \mathrm{NaCl}, 10 \mathrm{KCl}, 2 \mathrm{MgATP}$ and 0.1 Tris-GTP ( $\mathrm{pH} 7.25)$, was used.

Channelrhodopsin-expressing cells were imaged and recorded on an Olympus IX73 microscope with a 40x air objective equipped with Photometrics Cascade II 512 EMCCD camera. Images were acquired using Micromanager 1.4 with no gain and no electron multiplication and exposure time of $250 \mathrm{~ms}$. Excitation light was provided by a Thorlabs 4 channel LED (LED4D118 with 405, 470, 530 and $590 \mathrm{~nm}$ channels and only $470 \mathrm{~nm}$ and $530 \mathrm{~nm}$ light were used for imaging with LED driving currents of 150 and $80 \mathrm{~mA}$, respectively). Citrine and EYFP expression were imaged 
with a GFP filter cube (Semrock, FF02-470/30 excitation, FF01520/35 emission and FF495-Di03 dichroic) and tdTomato was imaged with a TRITC filter cube (Semrock, FF01-543/22 excitation, FF01-593/40 emission and FF562-Di03 dichroic).

For most channelrhodopsin stimulation experiments $10 \mathrm{~mW} /$ $\mathrm{mm} 2$ of $470 \mathrm{~nm}$ and $590 \mathrm{~nm}$ stimulation lights from Thorlabs 4 channel LED light source (LED4D118) were delivered to the specimen plane. The light intensity was controlled with a Thorlabs DC4104 4 channel controller electronically by the separate analogue outputs of a HEKA ITC-18 DAQ board under the control of WinWCP 5.5 software. In the spectral response experiment, a Sutter VF-5 system with a smart shutter in the neutral density mode was used to achieve cross spectrum stimulation at the same photon flux at $1.586 \times 1015$ photons / s / mm2 $(25.1 \mathrm{~mW} / \mathrm{mm} 2$ at $400 \mathrm{~nm}$ and $15.4 \mathrm{~mW} / \mathrm{mm} 2$ at $650 \mathrm{~nm}$ ) between $400-650$ $\mathrm{nm}(25 \mathrm{~nm}$ increments). The VF-5 has the following filters and bandwidth settings: 451-398 nm with $15 \mathrm{~nm}$ bandwidth, 503$446 \mathrm{~nm}$ with $15 \mathrm{~nm}$ bandwidth, 564-498 nm with $14 \mathrm{~nm}$ bandwidth, 632-557 nm with $14 \mathrm{~nm}$ bandwidth, 703-627 nm with $13 \mathrm{~nm}$ bandwidth. A second Sutter shutter was placed in the light path before the light guide to control the light output. For experiments with ACRs, a 15-sec interval was used between stimulation episodes. For ChrimsonR/IvfChrimson stimulation, stimulation light pulses either have 15 -sec intervals between stimulation episodes (no preconditioning) or a 15-sec interval - 1-sec $405 \mathrm{~nm}$ light pulse - 15-sec interval stimulation pattern for preconditioning before the subsequent stimulation. The stimulation light was guided into the fluorescent excitation light path of the Olympus IX73 microscope as with imaging but reflected with a Semrock FF685-DiO2 dichroic mirror to the objective.

Light intensity (in $\mathrm{mW} / \mathrm{mm} 2$ ) was measured with a Thorlabs slide based light detector (S170C) at the specimen plane with illumination area defined by the microscope aperture and manually calculated for photon flux or illumination intensity.

For comparison of the membrane expression of our IvfChrimson-citrine and ' $2 \mathrm{~A}$ ', transfected cells were imaged on an Olympus BX51 microscope with a 40x Olympus water immersion objective, X-Cite 110LED and Hamamatsu Orca Flash4. The images were taken at $15 \%$ light intensity and $250 \mathrm{~ms}$ exposure time with FF02-472/30 excitation filter, FF01-520/35 emission filter and FF495-Di03 dichroic mirror (Semrock, NY). A line profile was drawn across the cell and the membrane values were averaged and used for calculating the ratio with the mean cytosolic values.

\section{Organotypic slices and virus infection}

All procedures involving animals were approved by the Danish Animal Experiment Inspectorate. Hippocampi were isolated from postnatal P5-6 wild-type Sprague-Dawley rats (Janvier). The pups were decapitated and the brain removed and kept in an icy low sodium ACSF solution composed (in $\mathrm{mM}$ ) of $1 \mathrm{CaCl} 2,4 \mathrm{KCl}$, $1 \mathrm{MgCl} 2,26 \mathrm{NaHCO} 3,10$ D-Glucose, 234 Sucrose, and 0,1\% Phenol Red Solution, in mQ H2O, and bubbled with a mixture of CO2 (5\%) and O2 (95\%). From that moment, all the steps were performed in a laminar flow tissue culture hood using sterile equipment and in total asepsie. The brain was poured into a petri dish filled with the low sodium ACSF solution for hippocampi dissection under microscope guidance (Stereomicroscope, Olympus $\mathrm{x} 1$ objective). After extraction, the hippocampi were sliced at 400 um thickness using an automatic tissue chopper (McILWAIN) and moved to a dish with pre-heated culture medium containing MEM Eagle medium 78.8\% (Gibco), 20\% heat-inactivated horse serum (Gibco), $1 \mathrm{mM} \mathrm{L}$-glutamine, $1 \mathrm{mM} \mathrm{CaCl}$, $2 \mathrm{mM} \mathrm{MgSO} 4,170 \mathrm{nM}$ insulin, $0.0012 \%$ ascorbic acid, 12.9 $\mathrm{mM}$ D-glucose, $5.2 \mathrm{mM} \mathrm{NaHCO} 3,300 \mathrm{mM}$ HEPES (Sigma), $\mathrm{pH}=7.2-7.3$, osmolarity adjusted to 315-325. The slices with intact DG and CA regions were then transferred onto air-fluid interface-style Milli-cell culture inserts (Millipore) in 6-well culture plates (ThermoFisherScientific) with $800 \mathrm{uL}$ of sterile medium added below each insert. The slices were kept in a sterile incubator at 37 degrees with 5\% CO2 (ThermoScientific, Steri-cycle i-160). The medium was replaced by a pre-warmed medium (37 degrees) three times a week.

After two to three days of culture, the slices were microinjected in DG (or in CA1 when specified) along the characteristic horseshoe pattern with a pulled glass pipette containing the following viruses: retro-vfChrimson (titer: $7 \times 10^{12}$ particles $/ \mathrm{mL}$ ), retro-Zip(151T) (titer: 9x10 $10^{12}$ particles $/ \mathrm{mL}$ ), retro-ZipT-IvfChr (titer: $2 \times 10^{12}$ particles $/ \mathrm{mL}$ ), or retro-ZipV-IvfChr (titer: $1.5 \times 10^{12}$ particles $/ \mathrm{mL}$ ). The injections were done under a microscope (using a Picospitzer III (Parker) connected to a Pulse Pal (10 ms pulse, every 0.5-sec).

\section{Whole-cell electrophysiology and light delivery in-vitro}

Two to three weeks post-infection, the organotypic slices were transferred into the recording chamber and continuously perfused with Artificial Cerebrospinal Fluid containing (in mM): 119 $\mathrm{NaCL}, 2.5 \mathrm{KCl}, 26 \mathrm{NaHCO} 3,1 \mathrm{NaH} 2 \mathrm{PO} 4$, supplemented with $11 \mathrm{D}$-glucose, $4 \mathrm{CaCl}$, $4 \mathrm{MgCl}$. Additionally, APV (50mM), NBQX $(100 \mathrm{mM})$ and picrotoxin $(100 \mathrm{mM})$ were added to block excitatory and inhibitory fast transmission. The solution was adjusted to $\mathrm{pH} 7.4$ (osm -330) and bubbled with a mixture of CO2 $(5 \%)$ and $\mathrm{O} 2(95 \%)$, at room temperature. The chamber was mounted on an upright microscope (Scientifica) linked to a digital camera (QImaging Exi Aqua) and the cells were visualized using 60X water-immersion objective (Olympus, LumiPlan). Acquisitions were performed in whole-cell configuration using Clampex 10.6 connected to a Multiclamp 700B amplifier via a Digidata 1550A digitizer (all from Molecular Devices). Voltage-clamp data were low-pass filtered at $200 \mathrm{~Hz}$ and digitized at $10 \mathrm{kHz}$ and the 
whole-cell capacitance was compensated. Patch pipettes (2-4 M $\Omega$ of resistance) were filled with an internal solution containing (in $\mathrm{mM}$ ): $153 \mathrm{~K}$-gluconate, $10 \mathrm{HEPES}, 4.5 \mathrm{NaCl}, 9 \mathrm{KCl}, 0.6$ EGTA, 2 MgATP, and $0.3 \mathrm{NaGTP}$. The $\mathrm{pH}$ and osmolarity of the internal solution were close to physiological conditions $(\mathrm{pH}$ 7.4, osmolarity $297 \mathrm{mOsm}$ ). The access resistance of the cells in our sample was $-25 \mathrm{MOhm}$. For voltage-clamp experiment, the Dentate Gyrus granule cells (otherwise specified when done in CA1 pyramidal cells) were clamped at $-60 \mathrm{mV}$. For current-clamp experiments, the cells were maintained at $-60 \mathrm{mV}$ (except for the protocol where the potential was brought from -70 to $-40 \mathrm{mV}$ ) by constant current injection (holding current $\pm 100 \mathrm{pA}$ ).

The recorded neurons were illuminated with $470 \mathrm{~nm}$ and $635 \mathrm{~nm}$ (nominal wavelengths) light with a coolLED pE-4000 system connected to the Digidata via a single TTL. The light irradiance was controlled via the control pod and was adjusted to $4 \mathrm{~mW} /$ $\mathrm{mm} 2$ for $470 \mathrm{~nm}$ and $7 \mathrm{~mW} / \mathrm{mm} 2$ for $635 \mathrm{~nm}$ (otherwise specified). Light irradiance was measured with a Thorlabs digital optical power meter (PM100D) at the specimen plane with illumination area defined by the microscope aperture. Between the recordings, wavelengths were switched from $470 \mathrm{~nm}$ to $635 \mathrm{~nm}$ and vice versa. At the end of the recordings, the location of the recorded cell was confirmed by inspection at 10x magnification. Data analysis was performed using Clampfit 10.6 (Molecular device).

After recordings, slices were kept in $4 \%$ formalin, and later mounted under coverslips with Fluoromount (Sigma-Aldrich). Pictures were taken with an Apotome.2 (Zeiss) and ZEN software at 10X and 40X magnifications.

\section{Acute slices}

Experimental procedures were approved by the Animal Care and Use Committee of the University of Buenos Aires (CICUAL). Briefly, 4-week old C57 mice ( $\mathrm{n}=4$ ) were co-injected with AAV8/2-hSyn1-ChrimsonVF_EYFP_Kv2.1 and AAV-8/2-hSyn1ZipACR_Kv2.1_IRES_DsRed2 within the dentate gyrus of the hippocampus (250nl of a 1:1 mixture, coordinates from Bregma: $1 \mathrm{~mm}$ mediolateral, $-2 \mathrm{~mm}$ anteroposterior, -1.5 dorsoventral). After 3 weeks of expression, the animals were sacrificed and the brain removed and cut in a solution composed of (in $\mathrm{mM}$ ): 234 sucrose, 11 glucose, $26 \mathrm{NaHCO} 3,2.5 \mathrm{KCl}, 1.25 \mathrm{NaH} 2 \mathrm{PO}$, $10 \mathrm{MgSO} 4$, and $0.5 \mathrm{CaCl} 2$ (equilibrated with 95\% O2-5\% $\mathrm{CO} 2)$. The slices were maintained at room temperature before being transferred in a recording chamber mounted on a microscope (Nikon) connected to a Mightex Illumination system for $470 \mathrm{~nm}$ and $635 \mathrm{~nm}$ light delivery (at 10mW). For current clamp experiments the amount of current injected was corrected in the inter-sweep interval to keep it close to $-60 \mathrm{mV}$. In voltage clamp experiments holding potential was $-60 \mathrm{mV}$.

Recordings were done in a solution of composition (in $\mathrm{mM}$ ):
$126 \mathrm{NaCl}, 26 \mathrm{NaHCO} 3,2.5 \mathrm{KCl}, 1.25 \mathrm{NaH} 2 \mathrm{PO} 4,2 \mathrm{MgSO} 4,2$ $\mathrm{CaCl} 2$ and 10 Glucose (pH 7.4).

Patch-pipettes (2-4 M $\Omega$ of resistance) were filled with a K-gluconate based internal solution (in mM, $130 \mathrm{~K}$-gluconate, $5 \mathrm{KCl}, 10$ HEPES, 0.6 EGTA, 2.5 MgCl2.6H2O, 10 Phosphocreatine, 4 ATP-Mg, 0.4 GTP-Na3).

\section{Data Analysis}

The data were analysed and plotted using GraphPad Prism V9 (GraphPad Software, La Jolla, CA, USA). All values are indicated as mean \pm standard deviation (SD) or mean \pm standard error of the mean (SEM), as specified. For statistical comparison between groups, data were tested for normal distribution using D’Agostino and Pearson, Shapiro-Wilk or KS normality test. Comparison of the mean between groups has been done with a one-way analysis of variance (ANOVA) followed by Dunnett's multiple comparisons test.

ZEN software (Zeiss) and FIJI (from ImageJ) were used for images processing.

Figure 1A, 1B1 and 3E1 have been created with BioRender.com.

Acknowledgements. We thank the members of the Nabavi lab, in particular Islam Faress for suggestions, and Mariam Gamaleldin for the preparation of hippocampi organotypic slices. We also thank Dr. Sergio Almeida for his help on HEK293 cells culture at the early stage of the project, Peter Kerwin from the Philipsborn lab for his help on Drosophila work, and Anne-Katrine Vestergaard and Kathrine Meinecke Christensen for technical assistance. We are thankful to Dr. Thomas Mager and Dr. Ernst Bamberg for their generous gift of vfChrimson DNA vector. This study was supported by an ERC starting grant to S.N (22736), by the Danish Research Institute of Translational Neuroscience to S.N (19958), by PROMEMO (Center of Excellence for Proteins in Memory funded by the Danish National Research Foundation) to S.N (DNRF133), and by a Lundbeck NIH Brain Initiative grant to S. N and JY. L.

\section{References}

Bonaventura, J., Eldridge, M.A.G., Hu, F., Gomez, J.L., Sanchez-Soto, M., Abramyan, A.M., Lam, S., Boehm, M.A., Ruiz, C., Farrell, M.R., Moreno, A., Galal Faress, I.M., Andersen, N., Lin, J.Y., Moaddel, R., Morris, P.J., Shi, L., Sibley, D.R., Mahler, S.V., Nabavi, S., Pomper, M.G., Bonci, A., Horti, A.G., Richmond, B.J., Michaelides, M., 2019. High-potency ligands for DREADD imaging and activation in rodents and monkeys. Nat. Commun. 10, 4627. https://doi.org/10.1038/s41467-019-12236-z

Deisseroth, K., Hegemann, P., 2017. The form and function of channelrhodopsin. Science 357. https://doi.org/10.1126/science. aan5544

Ellendersen, B.E., von Philipsborn, A.C., 2017. 
Neuronal modulation of D. melanogaster sexual behaviour. Curr. Opin. Insect Sci., Neuroscience * Pheromones 24, 21-28. https:// doi.org/10.1016/j.cois.2017.08.005

Govorunova, E.G., Sineshchekov, O.A., Janz, R., Liu, X., Spudich, J.L., 2015. Natural light-gated anion channels: A family of microbial rhodopsins for advanced optogenetics. Science 349, 647-650. https://doi.org/10.1126/science.aaa7484

Govorunova, E.G., Sineshchekov, O.A., Rodarte, E.M., Janz, R., Morelle, O., Melkonian, M., Wong, G.K.-S., Spudich, J.L., 2017. The Expanding Family of Natural Anion Channelrhodopsins Reveals Large Variations in Kinetics, Conductance, and Spectral Sensitivity. Sci. Rep. 7, 43358. https://doi.org/10.1038/srep43358

Hooks, B.M., Lin, J.Y., Guo, C., Svoboda, K., 2015. Dual-Channel Circuit Mapping Reveals Sensorimotor Convergence in the Primary Motor Cortex. J. Neurosci. 35, 4418-4426. https://doi. org/10.1523/JNEUROSCI.3741-14.2015

Inagaki, H.K., Jung, Y., Hoopfer, E.D., Wong, A.M., Mishra, N., Lin, J.Y., Tsien, R.Y., Anderson, D.J., 2014. Optogenetic control of Drosophila using a red-shifted channelrhodopsin reveals experience-dependent influences on courtship. Nat. Methods 11, 325-332. https://doi.org/10.1038/nmeth.2765

Kato, H.E., Kim, Y.S., Paggi, J.M., Evans, K.E., Allen, W.E., Richardson, C., Inoue, K., Ito, S., Ramakrishnan, C., Fenno, L.E., Yamashita, K., Hilger, D., Lee, S.Y., Berndt, A., Shen, K., Kandori, H., Dror, R.O., Kobilka, B.K., Deisseroth, K., 2018. Structural mechanisms of selectivity and gating in anion channelrhodopsins. Nature 561, 349-354. https://doi.org/10.1038/s41586-0180504-5

Klapoetke, N.C., Murata, Y., Kim, S.S., Pulver, S.R., Birdsey-Benson, A., Cho, Y.K., Morimoto, T.K., Chuong, A.S., Carpenter, E.J., Tian, Z., Wang, J., Xie, Y., Yan, Z., Zhang, Y., Chow, B.Y., Surek, B., Melkonian, M., Jayaraman, V., Constantine-Paton, M., Wong, G.K.-S., Boyden, E.S., 2014. Independent Optical Excitation of Distinct Neural Populations. Nat. Methods 11, 338-346. https://doi.org/10.1038/nmeth.2836

Lin, J.Y., Knutsen, P.M., Muller, A., Kleinfeld, D., Tsien, R.Y., 2013. ReaChR: a red-shifted variant of channelrhodopsin enables deep transcranial optogenetic excitation. Nat. Neurosci. 16, 1499-1508. https://doi.org/10.1038/nn.3502

Lin, J.Y., Lin, M.Z., Steinbach, P., Tsien, R.Y., 2009. Characterization of engineered channelrhodopsin variants with improved properties and kinetics. Biophys. J. 96, 1803-1814. https://doi. org/10.1016/j.bpj.2008.11.034

Mager, T., Lopez de la Morena, D., Senn, V., Schlotte, J., D’Errico, A., Feldbauer, K., Wrobel, C., Jung, S., Bodensiek, K., Rankovic, V., Browne, L., Huet, A., Jüttner, J., Wood, P.G., Letzkus, J.J., Moser, T., Bamberg, E., 2018. High frequency neural spiking and auditory signaling by ultrafast red-shifted optogenetics. Nat. Commun. 9, 1750. https://doi.org/10.1038/s41467-018-041463

Mahn, M., Gibor, L., Patil, P., Cohen-Kashi Malina, K., Oring,
S., Printz, Y., Levy, R., Lampl, I., Yizhar, O., 2018. High-efficiency optogenetic silencing with soma-targeted anion-conducting channelrhodopsins. Nat. Commun. 9, 4125. https://doi. org/10.1038/s41467-018-06511-8

Mahn, M., Prigge, M., Ron, S., Levy, R., Yizhar, O., 2016. Biophysical constraints of optogenetic inhibition at presynaptic terminals. Nat. Neurosci. 19, 554-556. https://doi.org/10.1038/ nn. 4266

Marshel, J.H., Kim, Y.S., Machado, T.A., Quirin, S., Benson, B., Kadmon, J., Raja, C., Chibukhchyan, A., Ramakrishnan, C., Inoue, M., Shane, J.C., McKnight, D.J., Yoshizawa, S., Kato, H.E., Ganguli, S., Deisseroth, K., 2019. Cortical layer-specific critical dynamics triggering perception. Science 365. https://doi. org/10.1126/science.aaw5202

Mohammad, F., Stewart, J.C., Ott, S., Chlebikova, K., Chua, J.Y., Koh, T.-W., Ho, J., Claridge-Chang, A., 2017. Optogenetic inhibition of behavior with anion channelrhodopsins. Nat. Methods 14, 271-274. https://doi.org/10.1038/nmeth.4148

Vierock, J., Rodriguez-Rozada, S., Pieper, F., Dieter, A., Bergs, A., Zeitzschel, N., Ahlbeck, J., Sauter, K., Gottschalk, A., Engel, A.K., Hegemann, P., Wiegert, J.S., 2020. BiPOLES: a tool for bidirectional dual-color optogenetic control of neurons. bioRxiv 2020.07.15.204347. https://doi. org/10.1101/2020.07.15.204347

von Philipsborn, A.C., Liu, T., Yu, J.Y., Masser, C., Bidaye, S.S., Dickson, B.J., 2011. Neuronal control of Drosophila courtship song. Neuron 69, 509-522. https://doi.org/10.1016/j.neuron.2011.01.011

von Philipsborn, Anne C., Jörchel, S., Tirian, L., Demir, E., Morita, T., Stern, David L., and Dickson, Barry J. (2014). Cellular and Behavioral Functions of fruitless Isoforms in Drosophila Courtship. Curr Biol 24, 242-251. https://doi.org/10.1016/j. cub.2013.12.015

Waddell, W.H., Schaffer, A.M., Becker, R.S., 1973. Visual pigments. III. Determination and interpretation of the fluorescence quantum yields of retinals, Schiff bases, and protonated Schiff bases. J. Am. Chem. Soc. 95, 8223-8227. https://doi.org/10.1021/ ja00806a002

Wang, W., Nossoni, Z., Berbasova, T., Watson, C.T., Yapici, I., Lee, K.S.S., Vasileiou, C., Geiger, J.H., Borhan, B., 2012. Tuning the Electronic Absorption of Protein-Embedded All-trans-Retinal. Science 338, 1340-1343. https://doi.org/10.1126/science. 1226135

Yizhar, O., Fenno, L.E., Prigge, M., Schneider, F., Davidson, T.J., O'Shea, D.J., Sohal, V.S., Goshen, I., Finkelstein, J., Paz, J.T., Stehfest, K., Fudim, R., Ramakrishnan, C., Huguenard, J.R., Hegemann, P., Deisseroth, K., 2011. Neocortical excitation/inhibition balance in information processing and social dysfunction. Nature 477, 171-178. https://doi.org/10.1038/nature10360 
bioRxiv preprint doi: https://doi.org/10.1101/2021.05.05.442824; this version posted May 6, 2021. The copyright holder for this preprint (which was not certified by peer review) is the author/funder. All rights reserved. No reuse allowed without permission.

A

C-Chrimson-ts (HEK 293)

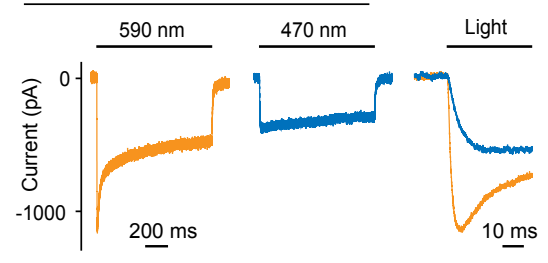

IvfChrimson (HEK 293)

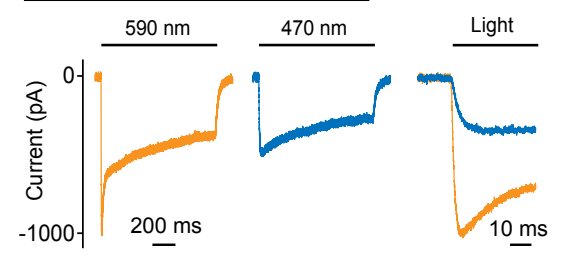

B

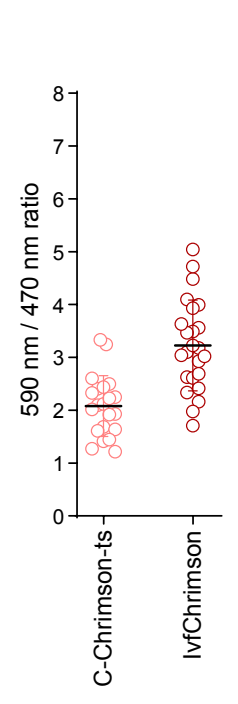

C

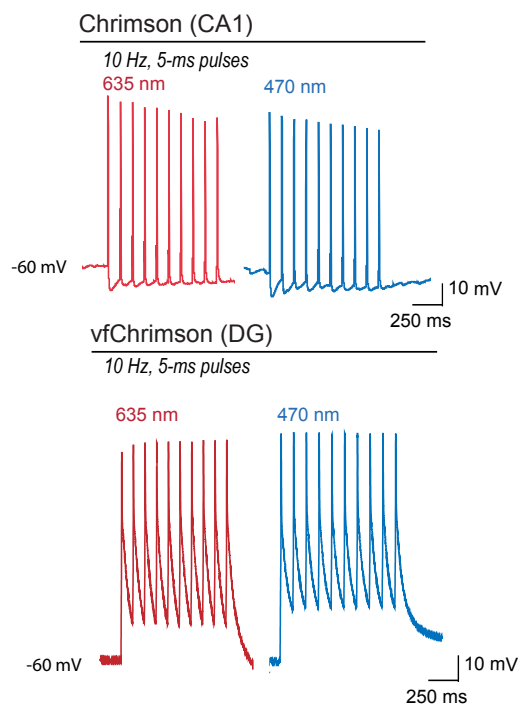

Supplementary figure 1. Red-shifted ChR2 variants are activated by $470 \mathrm{~nm}$ and $590 \mathrm{~nm}$ pulses of light. (A) Typical photocurrents (left and middle traces) and on-kinetics (right traces) of Chrimson-ts and IvfChrimson in HEK293 cells with $590 \mathrm{~nm}$ and $470 \mathrm{~nm}$ illumination at $10 \mathrm{~mW} / \mathrm{mm}^{2}$. The traces have been recorded 15 -s after a $405 \mathrm{~nm}$ reconditioning pulse. (B) Comparison of 590/470 nm ratio for Chrimson-ts and IvfChrimson, measured at peak. Each dot represents a cell (mean \pm SD). (C) Blue-light illumination of the Chrimson variants induces firing as efficient as red-light stimulation. This property is represented here with two examples of traces obtained from Chrimson expressing CA1 neurons and vfChrimson expressing DG neurons, in response to $10 \mathrm{~Hz}$ of $5 \mathrm{~ms}$ pulses at $635 \mathrm{~nm}$ and $470 \mathrm{~nm}$.

A

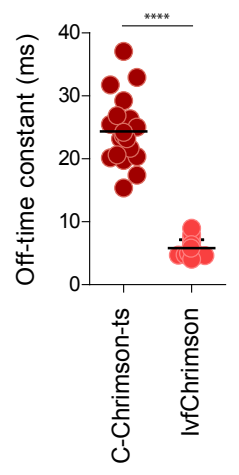

B

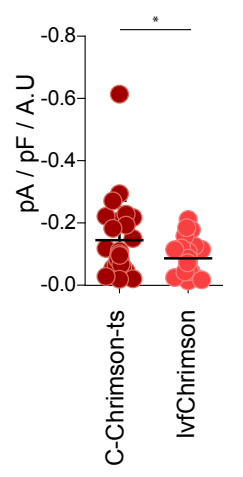

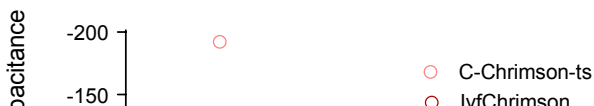

○ IvfChrimson

Supplementary figure 2. Membrane trafficking of the improved ultrafast vf-Chrimson, IvfChrimson. (A) Off-time constant of C-Chrimson-ts and IvfChrimson after 1-s of $635 \mathrm{~nm}$ illumination at $10 \mathrm{~mW} / \mathrm{mm}^{2}$. (B) Photocurrent amplitudes at the peak of C-Chrimson-ts and IvfChrimson, adjusted to membrane fluorescence (left), and comparison of current density and membrane fluorescence (right).

The recordings have been obtained from HEK293 cells at $-60 \mathrm{mV}$. Each dot represents the values from a single cell. The horizontal black bars represent the mean \pm SEM for (A) and mean \pm SD for (B). Statistics for panels (A) ${ }^{* * * *} \mathrm{p}<0.0001$; unpaired t-test and (B) ${ }^{*} \mathrm{p}<0.05$; unpaired t-test (Welch correction). 
bioRxiv preprint doi: https://doi.org/10.1101/2021.05.05.442824; this version posted May 6, 2021. The copyright holder for this preprint (which was not certified by peer review) is the author/funder. All rights reserved. No reuse allowed without permission.

A

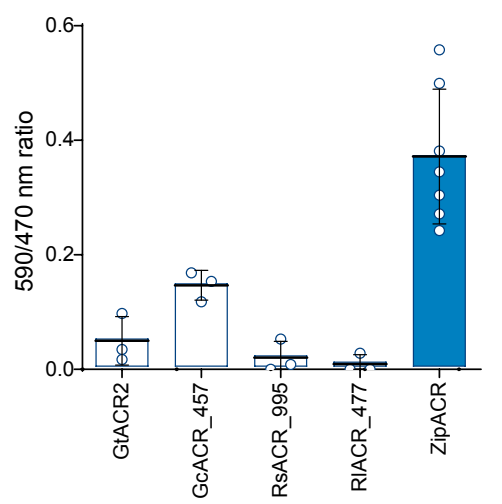

B

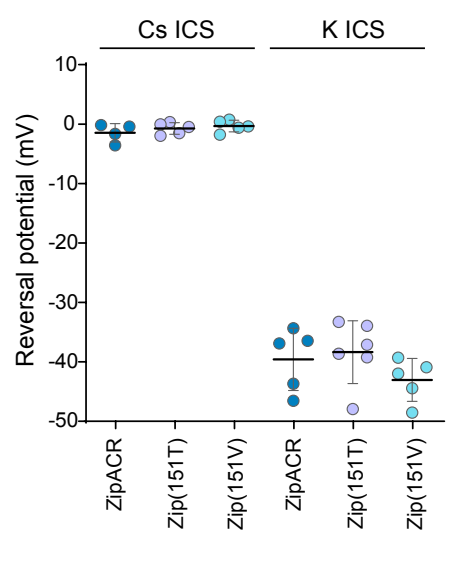

Supplementary figure 3. Channel properties of the ZipACR variants. (A) 590/470 ratio measured from the ACR variants in HEK293 cells. Each circle corresponds to value obtained from one cell (mean $\pm S D, n$ =3-7). (B) Reversal potential of the selected variants $\mathrm{Zip}(151 \mathrm{~T})$ and $\mathrm{Zip}(151 \mathrm{~V})$ compared to the original ZipACR with cesium (Cs) or potassium gluconate $(\mathrm{K})$ intracellular solution. Each dote corresponds to the value obtained from one cell (mean $\pm S D, n$ = 5-6). (C) Typical I/V-relationship for the two variants and ZipACR. The recordings have been done with- or without $470 \mathrm{~nm}$ LED illumination.

C

K-gluconate-based internal solution

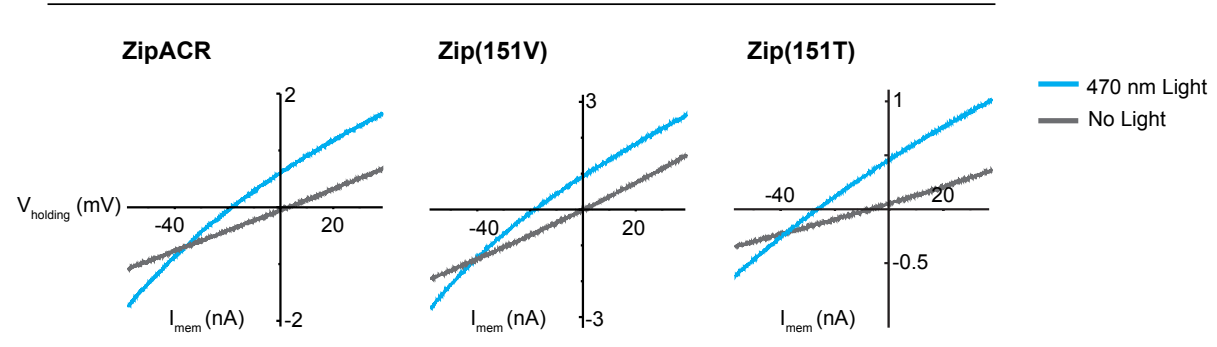


A

\section{ZipACR}

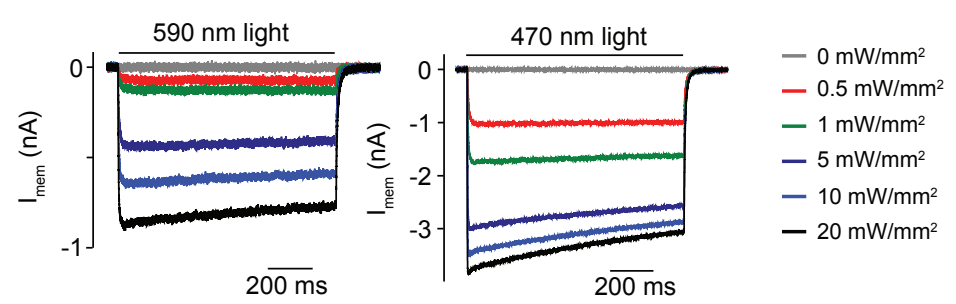

\section{Zip(I151V)}
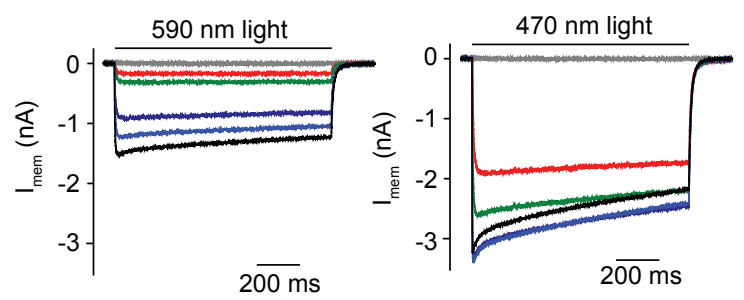

$470 \mathrm{~nm}$ light

\section{Zip(151T)}

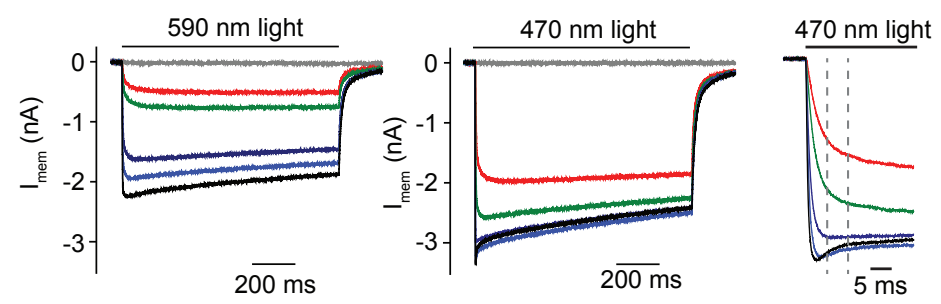

\section{B}
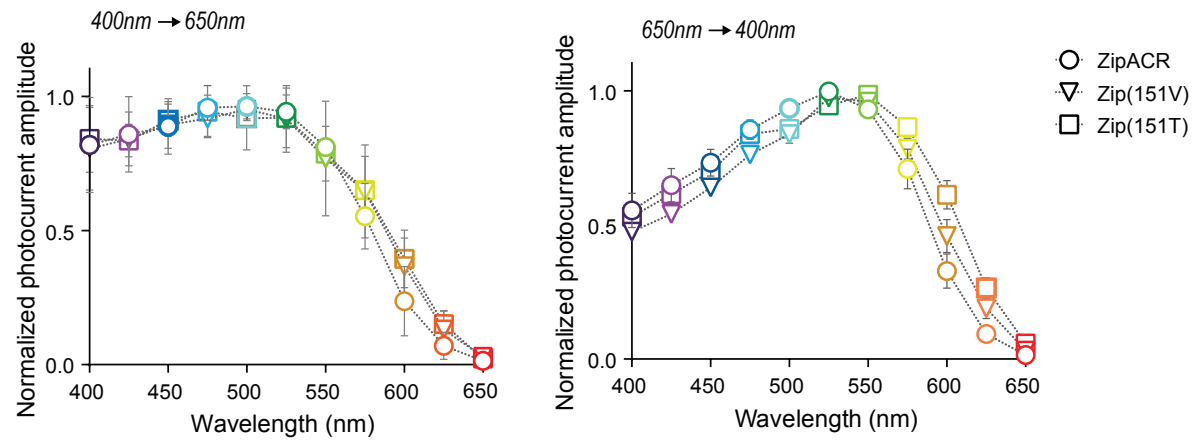

Supplementary figure 4. Photo-induced responses and action spectra of ZipACR variants. (A) Representative responses of the original ZipACR, Zip(151T) and Zip(151V) in HEK293 cells in response to different light intensities. (B) Normalized photocurrent amplitude in response to 400 nm-650 $\mathrm{nm}$ and to 650-400 nm light pulses (as presented in Fig. 3D2). The values have been measured on the photocurrents at the peak. The responses were normalized to the maximum response of each cell. The data points are the mean value \pm SEM $(n=6-7)$. 
bioRxiv preprint doi: https://doi.org/10.1101/2021.05.05.442824; this version posted May 6, 2021. The copyright holder for this preprint (which was not certified by peer review) is the author/funder. All rights reserved. No reuse allowed without permission.

A1

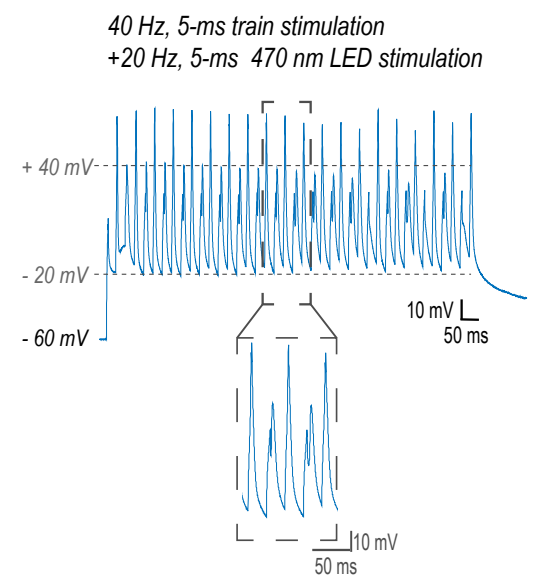

B1

$10 \mathrm{~Hz}, 5-m s 470 \mathrm{~nm}$ LED stimulation

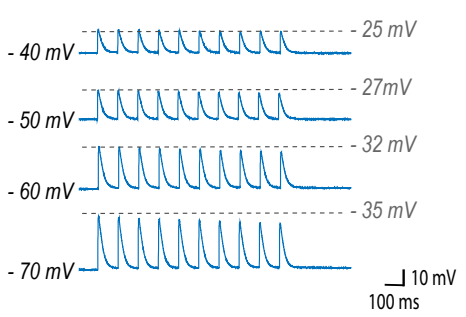

A2

$40 \mathrm{~Hz}, 5-m s$ train stimulation

+20 Hz, 5-ms 470 or $635 \mathrm{~nm}$ LED stimulation

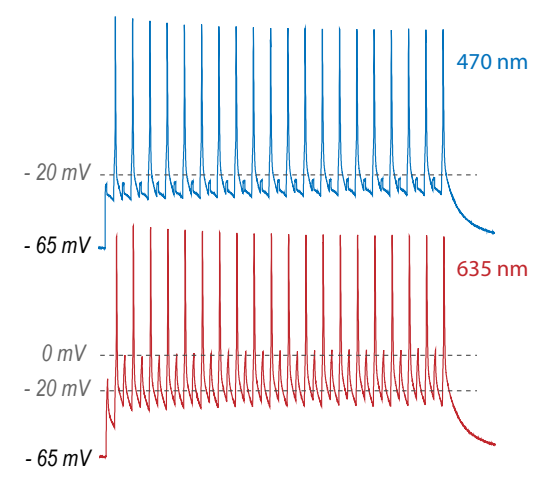

B2

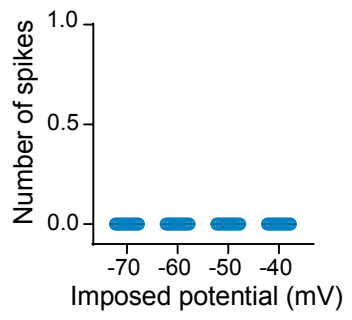

Supplementary figure 5. (A) The traces of the two outliers in Fig.3 E2. (A1), $470 \mathrm{~nm}$ illumination reduced the amplitude of the spikes, but did not abolish the firing. (A2), $470 \mathrm{~nm}$ illumination as well as $635 \mathrm{~nm}$ light blocked the firing. This cell expressed Zip(151T) channels which was significantly more red-shifted compared to the mean $(635 / 470 \mathrm{~nm}$ ratio: $23 \%$ for this cell vs $6 \%$ for the averaged population). (B) For a range of imposed potentials $470 \mathrm{~nm}$ illumination of $\mathrm{Zip}(151 \mathrm{~T})$ does not trigger action potentials. (B1) Typical traces of the DG neurons at various imposed potentials, in response to $10 \mathrm{~Hz}$ of $470 \mathrm{~nm}$ light illumination. The dotted lines show the potential of the maximal response. (B2) Plot showing the responses in current-clamp of the 10 neurons recorded at $-70,-60,-50$ and $-40 \mathrm{mV}$ imposed potential. Each dot represents a single cell. 
A
O ZipV-IvfChr

O IvfChrimson

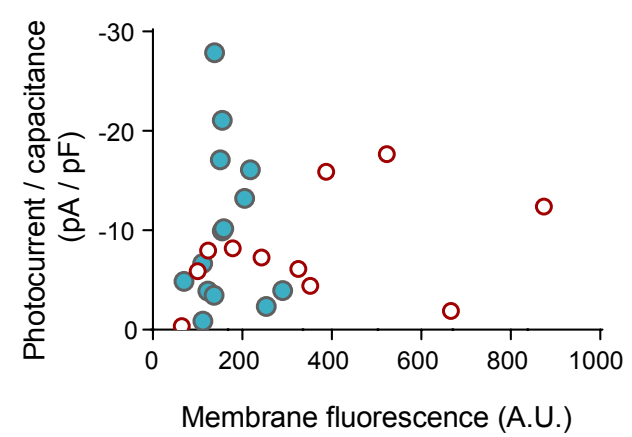

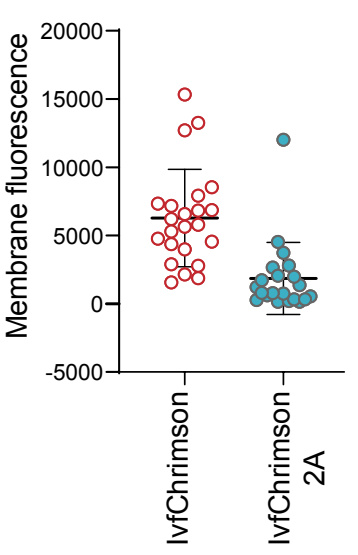

Supplementary figure 6. Improved membrane trafficking of vfChrimson. (A) Comparison of trafficking and photocurrent in HEK293 cells at $590 \mathrm{~nm}$ illumination for IvfChrimson and ZipV-IvfChr. (B) Comparison of the expression of IvfChrimson-citrine, and ' $2 \mathrm{~A}$ '. A line profile was drawn across the cell and the membrane values have been averaged and used for calculating the ratio with the mean cytosolic values. Each dot represents the value from a single cell $(\mathrm{n}=19-23$, mean \pm SD). (C) Representative pictures of HEK293 cells expressing the IvfChrimson or ' $2 \mathrm{~A}$ '. 
bioRxiv preprint doi: https://doi.org/10.1101/2021.05.05.442824; this version posted May 6, 2021. The copyright holder for this preprint (which was not certified by peer review) is the author/funder. All rights reserved. No reuse allowed without permission.

A

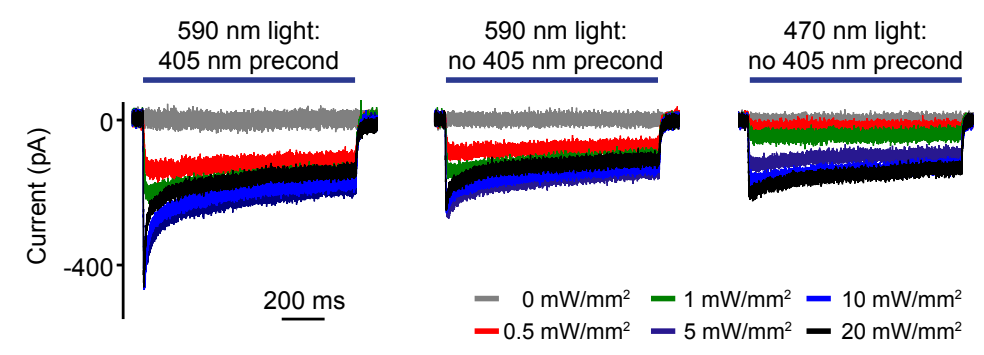

B

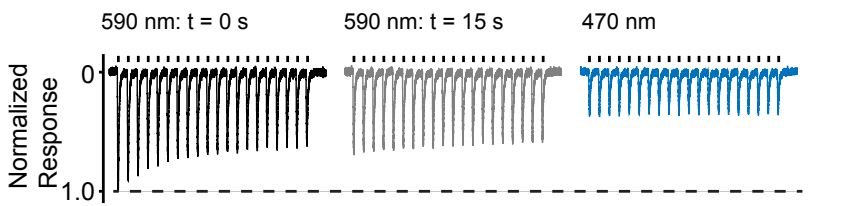

1st light pulse 20th light pulse
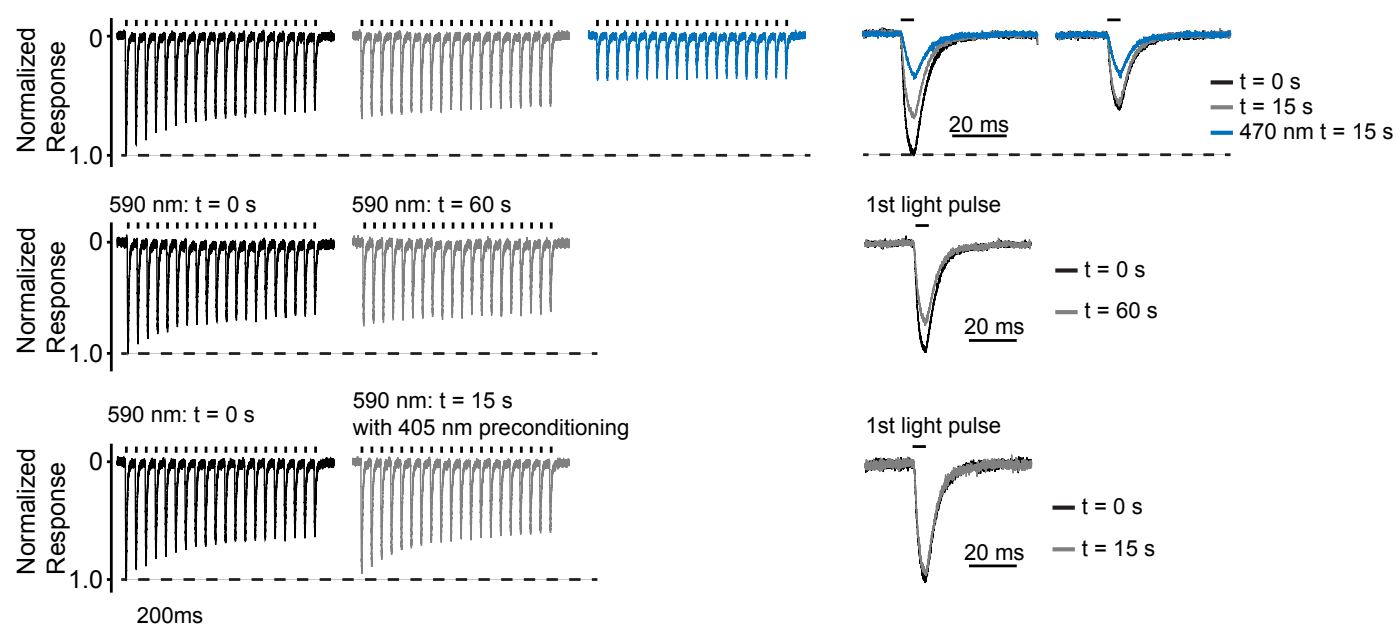

Supplementary figure 7. Excitation spectra of IvfChrimson in HEK293 cells. (A) Representative responses of IvfChrimson to 1 -sec of $470 \mathrm{~nm}$ and 590 $\mathrm{nm}$ with or without a preconditioning pulse at $405 \mathrm{~nm}$. (B) Normalized responses in voltage-clamp to $590 \mathrm{~nm}$ pulses of light, at $\mathrm{t}=0$, $\mathrm{t}=15 \mathrm{and} \mathrm{t}=60 \mathrm{sec}$ without $405 \mathrm{~nm}$ preconditioning. Due to desensitization IvfChrimson, responses do not recover at least within one minute. Prereconditioning pulse at 405 nm recovers the response. 
bioRxiv preprint doi: https://doi.org/10.1101/2021.05.05.442824; this version posted May 6, 2021. The copyright holder for this preprint (which was not certified by peer review) is the author/funder. All rights reserved. No reuse allowed without permission.

A

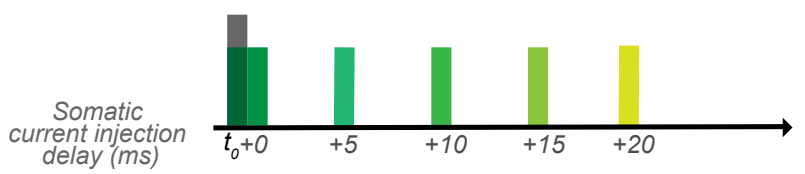

B
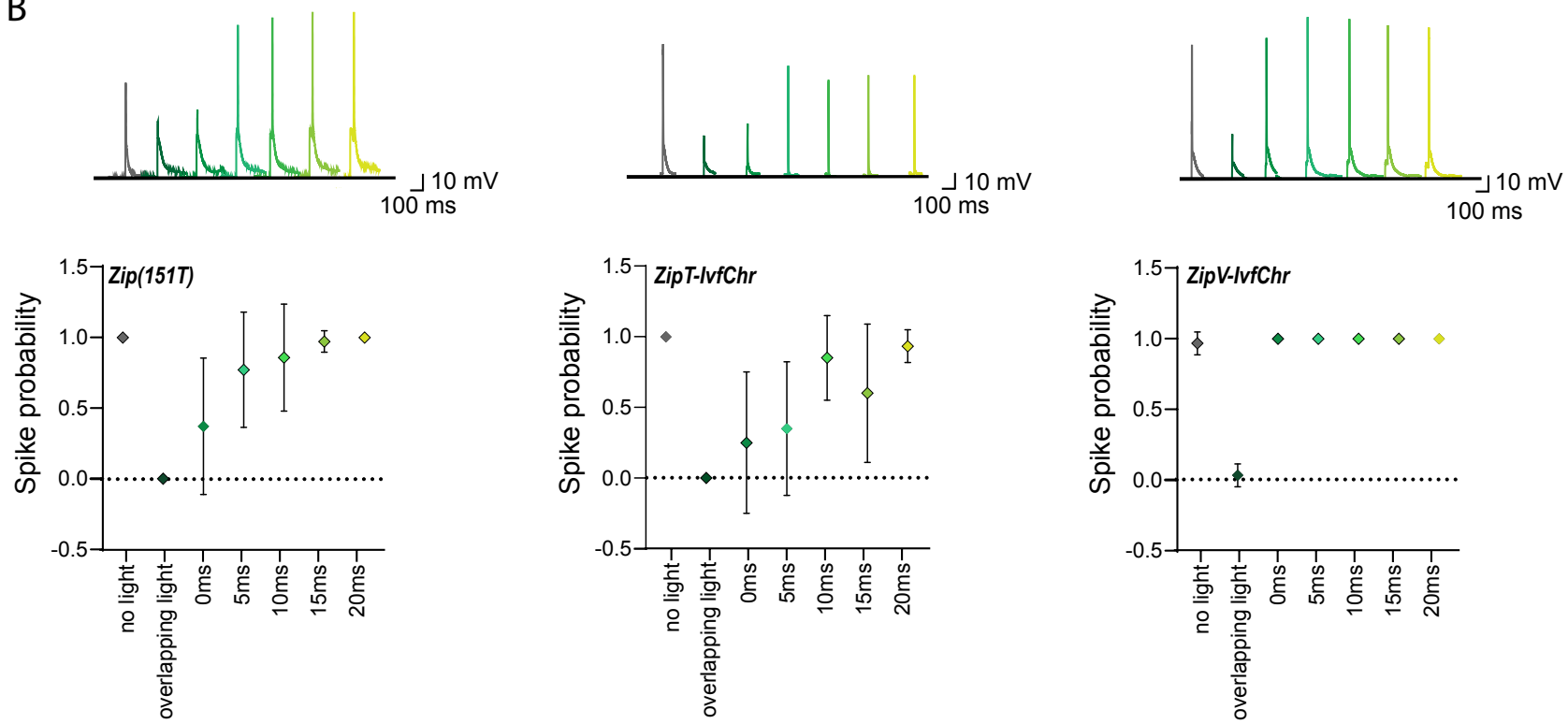

Supplementary figure 8. Temporal recovery of neuronal excitability following light-induced inhibition by the ZipACR variants. Immediate recovery of ZipV-IvfChr from the inhibition. (A) Experimental paradigm. DG granule cells expressing Zip(151T), ZipT-IvfCh or ZipV-IvfChr have been stimulated by a somatic current injection ( $5 \mathrm{~ms}$ pulse) overlapping or preceded by a $470 \mathrm{~nm}$ pulse $\left(5 \mathrm{~ms}, 4 \mathrm{~mW} / \mathrm{mm}^{2}\right)$ at $0,5,10,15$ or $20 \mathrm{~ms}$ intervals. (B) The traces in current-clamp (top) are representative of the responses to current injection for the three constructs. The spike probability (bottom) shows an immediate recovery of ZipV-IvfChr from the off-set of the $470 \mathrm{~nm}$ light ( $\mathrm{n}=7$ per construct, mean \pm SD). 